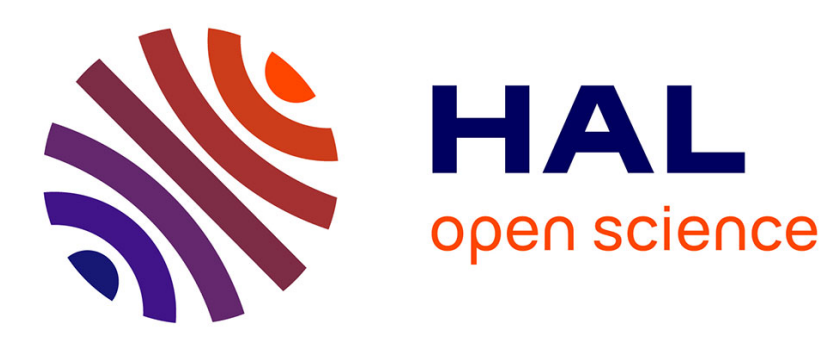

\title{
A general BV existence result for conservation laws with spatial heterogeneities
}

\author{
Benedetto Piccoli, Magali Tournus
}

\section{To cite this version:}

Benedetto Piccoli, Magali Tournus. A general BV existence result for conservation laws with spatial heterogeneities. SIAM Journal on Mathematical Analysis, 2018, 50 (3), pp.2901-2927. 10.1137/17M112628X . hal-01352538v3

\section{HAL Id: hal-01352538 \\ https://hal.science/hal-01352538v3}

Submitted on 6 Apr 2018

HAL is a multi-disciplinary open access archive for the deposit and dissemination of scientific research documents, whether they are published or not. The documents may come from teaching and research institutions in France or abroad, or from public or private research centers.
L'archive ouverte pluridisciplinaire HAL, est destinée au dépôt et à la diffusion de documents scientifiques de niveau recherche, publiés ou non, émanant des établissements d'enseignement et de recherche français ou étrangers, des laboratoires publics ou privés. 


\title{
A general BV existence result for conservation laws with spatial heterogeneities
}

\author{
Benedetto Piccoli * $\quad$ Magali Tournus ${ }^{\dagger}$
}

\begin{abstract}
We consider a scalar conservation law with a flux containing spatial heterogeneities of bounded variation, where the number of discontinuities may be infinite. We prove existence of an adapted entropy solution in the sense of Audusse-Perthame in the BV framework, under the assumptions that guarantee uniqueness of the solution, plus one additional technical assumption. We prove that existence in the BV framework fails withouth this additional technical assumption, by providing a counter-example.
\end{abstract}

Key-words. Scalar conservation laws, Discontinuous flux with Bounded Variations, FrontTracking Method, Adapted entropies.

AMS subject classifications. 35L65, 35B44, 35A01.

\section{Introduction}

We are interested in scalar conservation laws with a flux containing spatial heterogeneities. The conservation law is written as

$$
\left\{\begin{aligned}
\frac{\partial \rho}{\partial t}+\frac{\partial}{\partial x} A(\rho, x) & =0, \quad t>0, x \in \mathbb{R} \\
\rho(0, x) & =\rho_{0}(x), \quad x \in \mathbb{R}
\end{aligned}\right.
$$

where $A(\rho,$.$) may a priori be discontinuous in an infinite number of points x$, and where $\rho_{0} \geq 0$.

There are many models that lead to consider hyperbolic conservation laws with a flux function discontinuous in the state space, arising in fields as various as vehicular traffic flows [5] [14] [20], two-phase flows porous medias [3] (see also [19]), sedimentation [13], kidney physiology [29], cell dynamics [8], and others.

\subsection{Two selection criteria for general fluxes}

When the space dependence is sufficiently smooth, well-posedness of the scalar equation (1) is wellknown and the commonly admitted criterion to select a physical solution was described by Kružkov [25. A function $\rho \in L^{\infty}\left(\mathbb{R}^{+} \times \mathbb{R}\right)$ is an entropy solution of (1) if for all $k \in \mathbb{R}$ it satisfies in the distribution sense that

$$
\frac{\partial}{\partial t}|\rho-k|+\frac{\partial}{\partial x}\left(\operatorname{sign}(\rho-k)(A(\rho, x)-A(k, x))+\operatorname{sign}(\rho-k) \frac{\partial}{\partial x} A(k, x) \leq 0 .\right.
$$

${ }^{*}$ Department of Mathematical Sciences, Rutgers University Camden, Camden, NJ, USA. Email: piccoli@camden.rutgers.edu.

${ }^{\dagger}$ Aix Marseille Univ, CNRS, Centrale Marseille, I2M, Marseille, France. Email: mtournus@math.cnrs.fr. 
For discontinuous fluxes, formulation (2) does not make sense: Since weak solutions of (1) satisfy the Rankine-Hugoniot conditions through the discontinuities $x_{0}$, i.e. $A\left(\rho\left(x_{0}^{-}, t\right), x_{0}^{-}\right)=$ $A\left(\rho\left(x_{0}^{+}, t\right), x_{0}^{+}\right)$, the points $x_{0}$ where $A$ is discontinuous are precisely the points where $\rho(., t)$ is discontinuous. Thus, the product $\left(\operatorname{sign}(\rho-k) \frac{\partial}{\partial x} A(k, x)\right)$ which appears in (2) may not be defined. Different criteria were then introduced to select the appropriate weak solution. Contrary to the case of Lipschitz continuous flux, there is no general agreement on the right concept of entropy solution for discontinuous fluxes: the solution we wish to select may depend on the context.

In the last two decades, different criteria for different contexts were proposed. In most theories, authors restrict to fluxes with separated variables, i.e. of the type $A(\rho, x)=a(\rho) f(x)$, see [4], [9], [31], where $f$ has a finite number of discontinuities, usually only one. Uniqueness theory then mostly relies on some given constraints imposed on the solution at the points where the flux is discontinuous, whereas, elsewhere, the usual entropy formulation holds, see [1], [2], [12]. A natural condition to impose, at the points $x$ where the flux is discontinuous, is the continuity of the flux through the heterogeneity

$$
A\left(\rho\left(x^{-}, t\right), x^{-}\right)=A\left(\rho\left(x^{+}, t\right), x^{+}\right), \quad t>0,
$$

but it is not sufficient to isolate a unique solution. Interface entropy conditions may depend on which solution we aim to select and may thereby vary with the context. Notice that to impose specific entropy conditions at discontinuities of $f$, the use of traces across this line [33] is mandatory, which requires the solution to be regular enough.

The existence of solutions is then usually obtained either by using specific Riemann solvers related to the entropy formulation ([15], 21], 24]), either by approximating the flux by regular ones ([22]) or by passing to the limit along robust and convergent numerical schemes, sometimes established independently of any entropy criterion ([23], [32]). Note that the determination of the appropriate Riemann solver is a necessary tool to define a Godunov scheme [27]. In all of the works cited above, in addition to the separated variable framework, strong modeling assumptions were done regarding the flux $a$ (convexity, concavity, monotony, ...) and on the initial conditions $\rho_{0}$.

One can find in [6] an exhaustive review of selection criterions available in the literature of discontinuous fluxes in the case

$$
A(\rho, x)= \begin{cases}a_{-}(\rho), & x<0, \\ a_{+}(\rho), & x>0,\end{cases}
$$

where $a_{-}$and $a_{+}$are Lipschitz continuous functions. Each existing admissibility condition (i.e. each selection criterion that leads to a $L^{1}$-contractivity property) is associated with a germ that underly these conditions.

In the present paper, we only focus on formulations that allow an infinite number of discontinuities, with possibly an accumulation point. Among the numerous interface conditions introduced, two of them were extended to general fluxes (by "general fluxes", we mean fluxes $A$ that are not written $A(\rho, x)=a(\rho) f(x))$. In both formulations, the idea is to remove the last term in (2). A common feature to these two formulations is the continuity of the flux through discontinuities given by the Rankine-Hugoniot condition. Both selection criteria are purely mathematical criteria.

\section{Audusse-Perthame adapted entropy formulation}

The theory for conservation laws with BV flux we consider in this paper was introduced by Baiti-Jenssen [10] in 1997, and then developed by Audusse-Perthame [7] in 2005. The novelty of the approach of Baiti-Jensen [10] is that their framework does not distinguish the discontinuity points one by one, allowing them to be infinite: The entropy criterion is not written in term of 
interface condition. Their idea is to modify the usual Kružkov entropy formulation, which makes no sense at the points where the flux is discontinuous, and to adapt the entropies to the discontinuous flux. Here also, the theory provides a framework in which the solution of (1) can be uniquely defined provided that the flux $A$ satisfies the following assumptions

A-1. For all $\rho \in \mathbb{R}^{+}, x \mapsto A(\rho, x)$ is BV,

A-2. There are two continuous functions $A_{-} \geq 0, A_{+} \geq 0$ (with $A_{ \pm}(\rho)>0$ for $\rho>0$ ), such that $A( \pm \infty)=+\infty$, and $A_{-}(\rho) \leq|A(\rho, x)| \leq A_{+}(\rho)$ for all $x \in \mathbb{R}$.

A-3. For almost all $x \in \mathbb{R}, A(., x)$ is locally Lipschitz and one-to-one.

Under hypotheses (A-1.) (A-2.) (A-3.), the usual Kružkov [25] entropies $(|\rho-k|)_{k \in \mathbb{R}}$ are replaced by the adapted entropies $\left(\left|\rho-k^{p}(x)\right|\right)_{p \in \mathbb{R}}$, where $k^{p}$ is the unique solution of

$$
A\left(k^{p}(x), x\right)=p \quad \text { a.e. } x \in \mathbb{R} .
$$

The existence and uniqueness of the $k^{p}(x)$, defined in (3), is guaranteed by the fact that $A(., x)$ is one-to-one. Let us notice that the functions $k^{p}$ are the solutions to the stationary problem

$$
\frac{d}{d x} A\left(k^{p}(x), x\right)=0 .
$$

When $A$ does not depend on $x$, the functions $k^{p}$ are then constant, as in the classical Kružkov formalism. The surjectivity of $A(., x)$ into the space $\operatorname{Im}(A)$ which does not depend on $x$ (in (A-3.), $\operatorname{Im}(A)=\mathbb{R})$ is crucial to guarantee the existence of the adapted entropies associated with (1) and also to guarantee the existence of a solution to the Riemann problem. However, the injectivity may be relaxed. Indeed, assumption A-3. can be replaced by

A-4. There exists a function $u$ such that for all $x \in \mathbb{R} \backslash N, A(., x)$ is a locally Lipschitz one-to-one function from $(-\infty, u(x)]$ and $[u(x),+\infty)$ to $[0,+\infty)$ such that $A(u(x), x)=0$.

Given $p \geq 0$, there are two unique functions $k^{p,-}(x) \in(-\infty, u(x)]$ and $k^{p,+}(x) \in[u(x),+\infty)$ such that $A\left(k^{p, \pm}(x), x\right)=p$. This framework leads to the following definition of entropy solutions :

Definition 1 (Adapted Entropy solution). A function $\rho \in L^{\infty}([0, T] \times \mathbb{R}) \cap \mathcal{C}^{0}\left([0, T], L_{\text {loc }}^{1}(\mathbb{R})\right)$ is an adapted entropy solution of (1), on $[0, T] \times \mathbb{R}$ if for all the $\left(k^{p}\right)_{p \in \mathbb{R}}$ satisfying (3) (or in case (A-4.), for all $k^{p,+}, k^{p,-}$ ), the following inequality holds in the sense of distributions

$$
\frac{\partial}{\partial t}\left|\rho(x, t)-k^{p}(x)\right|+\frac{\partial}{\partial x}\left\{\operatorname{sign}\left(\rho(x, t)-k^{p}(x)\right)\left[A(\rho(x, t), x)-A\left(k^{p}(x), x\right)\right]\right\} \leq 0 .
$$

This formulation is well-adapted to guarantee uniqueness and $L^{1}$ stability with respect to the initial condition, since the technique of doubling the variables can be applied. However, existence of such an entropy solution for (1) has only been shown under the specific assumption

$$
A(\rho, x)=F(\rho, v(x)),
$$

where $v \in \mathrm{BV}(\mathbb{R})$ and $F \in \mathcal{C}^{2}\left(\mathbb{R}^{2}\right)$, with the problem of existence for a general flux still open and explored in the present paper. We also mention that contrary to the Panov formulation, extending Audusse-Perthame adapted entropies to multi-dimensional conservation laws is still an 
open problem. To the best of our knowledge, writing a numerical scheme to solve this formulation is an open problem as well.

Panov formulation Another formulation was initiated by Klingenberg and Risebro [24] who selected a weak solution shown to be unique and stable under a wave entropy condition. Based on this idea, Towers [32] then defined a notion of entropy solution and proved the uniqueness of piecewise smooth entropy solutions. The definition of Towers is a global definition in the sense that the entropy conditions are enclosed in the weak formulation and not required as local conditions. The theory only deals with fluxes of type $A(\rho, x)=a(x) g(\rho)$, with $g(\rho)=\rho(1-\rho)$, and with $a(x)=a_{L}$ for $x<0$, and $a(x)=a_{R}$ for $x>0$. For $\rho_{0} \in L^{\infty}(\mathbb{R})$ such that $0 \leq \rho_{0} \leq 1$, a function $\rho \in L^{\infty}\left(\mathbb{R}^{+} \times \mathbb{R}\right)$ is an entropy solution if for all $k \in \mathbb{R}$ it satisfies in the sense of distributions

$$
\frac{\partial}{\partial t}|\rho(x, t)-k|+\frac{\partial}{\partial x}(\operatorname{sign}(\rho-k)(A(\rho, x)-A(k, x)))+\left|a_{L}-a_{R}\right| g(k) \delta_{0} \leq 0 .
$$

A motivation to define such a criterion is the following: let us approximate $a$ by a sequence of smooth monotonous functions $a_{\varepsilon}$. Using (2), a unique sequence of functions $\rho_{\varepsilon}$ can then be defined so that they satisfy

$$
\frac{\partial}{\partial t}\left|\rho_{\varepsilon}-k\right|+\frac{\partial}{\partial x}\left(\operatorname{sign}\left(\rho_{\varepsilon}-k\right) a_{\varepsilon}(x)\left(g\left(\rho_{\varepsilon}\right)-g(k)\right)\right)+\operatorname{sign}\left(\rho_{\varepsilon}-k\right) a_{\varepsilon}^{\prime}(x) g(k) \leq 0 .
$$

It is proved in [31] that the sequence $\rho_{\varepsilon}$ converges strongly toward $\rho \in L^{\infty}\left(\mathbb{R}^{+} \times \mathbb{R}\right)$. Since for all $k \in \mathbb{R}$ we have

$$
\operatorname{sign}\left(\rho_{\varepsilon}-k\right) a_{\varepsilon}^{\prime}(x) g(k) \leq\left|a_{\varepsilon}^{\prime}(x)\right| g(k) \underset{\varepsilon \rightarrow 0}{\longrightarrow}\left|a_{L}-a_{R}\right| g(k) \delta_{0}
$$

the unique function $\rho$ selected by (6) is the limit of the sequence $\rho_{\varepsilon}$. Existence of solutions is obtained in [32] through the convergence of a Godunov scheme, by using a discretization of $a$ staggered with respect to that of $\rho$ and proving BV estimates. Notice that the Riemann solver associated with this formulation may not be intuitive. It may happen that at the interface, 3 waves are created by the discontinuity (see example b) below).

This formulation has been extended to the framework of general fluxes (non-separated variables) in [28. The concavity assumption was removed, but the non-degeneracy of the flux (see assumption (B-2.) below) is crucial to guarantee the existence of traces [33. The theory provides a framework in which the solution of (1) can be defined provided that the flux $A$ and the initial condition $\rho_{0}$ satisfy for some $\left(\rho_{m}, \rho_{M}\right) \in \mathbb{R}^{2}$,

B-1. $\rho_{m} \leq \rho_{0}(x) \leq \rho_{M}$ and $A\left(\rho_{m}, x\right)=A\left(\rho_{M}, x\right)=C$ for some $C \in \mathbb{R}$,

B-2. The flux is not linearly-degenerate in the sense that for all $(\xi, \zeta) \in \mathbb{R}^{2} \backslash\{(0,0)\}$, for all $\rho \geq 0$, $\mu\left\{x: \zeta+\xi \frac{\partial A}{\partial \rho}(\rho, x)=0\right\}=0$, where $\mu$ denotes the one dimensional Lebesgue measure.

B-3. For all $\rho \in\left[\rho_{m}, \rho_{M}\right], x \mapsto A(\rho, x)$ is $\mathrm{BV}$, and $\max _{\rho \in\left[\rho_{m}, \rho_{M}\right]} A(., \rho) \in L_{l o c}^{q}(\mathbb{R}), q>2$.

We notice here that $(\mathrm{A}-3$.$) and (\mathrm{B}-1$.$) are mutually exclusive, so that the two theories cannot be$ compared.

Definition 2 (Panov Solution). Assume that the flux A satisfies (B-1.) (B-2.) (B-3.). For any $k \in \mathbb{R}$, call $\mu_{k}^{c}$, respectively $\mu_{k}^{s}$, the continuous, respectively singular, part of the distributional derivative of the map $x \mapsto A(k, x)$. A function $\rho \in L^{\infty}\left(\mathbb{R}^{+} \times \mathbb{R}\right)$ is a Panov solution to (1) if for any $k \in \mathbb{R}$

$$
\frac{\partial}{\partial t}|\rho-k|+\frac{\partial}{\partial x}(\operatorname{sign}(\rho-k)(A(\rho, x)-A(k, x)))+\operatorname{sign}(\rho-k) \mu_{k}^{c}(x)+\left|\mu_{k}^{s}(x)\right| \leq 0 .
$$


We stated here Definition 2 in one space dimension, but the existence result is stated in [28] for multi-dimensional conservation laws. Existence is obtained through the weak convergence of measure-valued functions and pre-compactness in specific Sobolev spaces. However, uniqueness was not proven in that extended context. Recently, the authors of [26] proved existence without assumption B1. The two main differences with our result are that they assume a non degeneracy condition on the flux (assumption 4.4 of their paper), whereas we can deal with piecewise-linear fluxes, and that there are some fluxes (for instance the one presented in Section 1.2-a) for which both Lazar and Mitrovi have existence, but the solution selected is not the same. Numerical simulations were performed [31] using Godunov type schemes.

We end this section with a list of comparative examples between these two formulations.

\subsection{Comparison between the two formulations}

Both formulations described above were introduced for different purposes, and are adapted to different types of fluxes. Other formulation can also be found in the litterature, for instance in [16] [17] where the authors introduce an entropy criterion based on the two criteria mentioned here and obtain uniqueness (but not existence) of BV entropy solutions. We provide here some examples to compare the two formulations.

a) Both formulations are defined but the solutions do not coincide. For the following flux and initial condition,

$$
A(\rho, x)=H(x) \frac{\rho^{2}}{2}+(1-H(x)) \frac{(\rho-1)^{2}}{2}, \quad \rho_{0}(x)=\frac{1}{2},
$$

where $H$ denotes the usual Heaviside function, the two formulations select distinct solutions. Actually, this flux does not satisfy the assumption (B-1.). However, for any $M$ large enough, we can replace it by a flux $A_{M}$ which coincides with $A$ over $[-M, M]$, and which satisfy (B-1.) (B-2.) (B-3.), with $A_{M}(M+1, x)=A_{M}(-(M+1), x)$. One can prove (see Appendix 2) that for any $M$ large enough, the Panov definition selects the constant solution $\rho(x, t)=\frac{1}{2}$. On the other hand, the Audusse-Perthame (see [7]) solution is composed of a rarefaction wave $(1 / 2,1)$ moving to the left, a stationary shock $(1,0)$ localized at $x=0$ and another rarefaction wave $(0,1 / 2)$ moving to the right. More precisely,

$$
\rho(x, t)=1+\frac{x}{t} \text { for }-\frac{t}{2}<x \leq 0, \quad \rho(x, t)=\frac{x}{t} \text { for }<0 \leq \frac{t}{2}, \quad \text { and } \rho(x, t)=\frac{1}{2} \text { elsewhere. }
$$

b) Only Panov solution is defined. The following flux does not satisfy the Audusse-Perthame assumptions

$$
A(\rho, x)=a(x) \rho(1-\rho), \quad a(x)=\left\{\begin{array}{ll}
16, & x<0, \\
1, & x>0,
\end{array} \quad \rho_{0}(x)=\frac{1}{4} .\right.
$$

The Panov solution is composed of three waves: A shock $\left(\frac{1}{4}, \frac{1}{2}+\frac{\sqrt{15}}{8}\right)$ moving to the left, a stationary shock $\left(\frac{1}{2}+\frac{\sqrt{15}}{8}, \frac{1}{2}\right)$ localized at the discontinuity point $x=0$, and a rarefaction wave $\left(\frac{1}{2}, \frac{1}{4}\right)$ moving to the right. 
c) Only Audusse-Perthame solution is defined. The following linearly-egenerate flux is not included in Panov formulation

$$
A(\rho, x)=H(x)|\rho|+(1-H(x))|\rho-1|, \quad \rho_{0}(x)=\frac{1}{2} .
$$

The Audusse-Perthame solution is composed of a contact discontinuity $(1 / 2,1)$ moving to the left, a stationary shock $(1,0)$ localized at $x=0$ and another contact discontinuity $(0,1 / 2)$ moving to the right.

d) None of the formulations is defined. Let $f$ be strictly increasing and $g$ be strictly decreasing, and define the flux

$$
A(\rho, x)= \begin{cases}f(\rho), & x<0 \\ g(\rho), & x>0\end{cases}
$$

This flux does not enter neither Panov, nor Audusse-Perthame formulation. When fluxes have opposite monotonicity on each side of the discontinuity, either the solution is a measure: a delta function appears at the discontinuity points of the flux (singular compressive case) so that a BV theory is hopeless, or the problem is ill-posed whatever the framework (singular expansive case). See for instance the linear case $A(\rho, x)=\rho \operatorname{sign}(x)$ detailed in [30]: whatever the definition of the solution, it is not possible to build a theory for which stability with respect to initial condition holds.

In order to stress the importance of the surjectivity assumption in the Audusse-Perthame formulation, we provide another example of a flux and initial condition for which no formulation can be applied. Let us define

$$
A(\rho, x)=\left\{\begin{array}{l}
e^{\rho}, \quad x<0 \\
2+e^{\rho}, \quad x>0, \quad \rho_{0}(x)=0 .
\end{array}\right.
$$

In that case also, there is no right notion of weak solution: For $x<0$, wave can only propagate from left to right, and the continuity of the flux at the interface prevents the existence of any admissible value for the trace of the solution at $x=0^{+}$.

\section{Main Results}

Audusse-Perthame formulation has been set in order to deal with discontinuous fluxes of general shape, possibly having an infinite number of discontinuities. The formulation is not based on the solution of interface problems: the selection process is a pure mathematical criterion. It was proved [7] that the criterion was enough to select a unique solution. However, the question of general existence is not solved. We explore here the question of existence of Audusse-Perthame solutions in the BV framework. To do so, we identify what are the key-assumptions on the flux which are necessary to build this theory, and provide in that sense optimal general estimates on the total variation of the solution. We prove that there exists a solution that can be built via solving Riemann problems for an approximated system, and then passing the sequence of solutions to the limit via the Wave Front-Tracking method, proving stability of the solution with respect to the flux. The function we obtain at the limit coincides with the adapted entropy solution (Definition 1). This result is a generalization of [10] in the sense that we consider fluxes not included in their framework.

\subsection{Framework of the paper}

We set here the assumptions we make about the regularity and the shape of the flux. For modeling purposes and simplicity of exposition, we adopt the following framework 
$\mathrm{H}-0 \rho \mapsto A(\rho, x)$ is concave, strictly increasing, and $A(0, x)=0$, for every $x \in \mathbb{R}$.

H-1 there exist constants $c>0, C>0$, not depending on $\rho$ or $x$, and a continuous function $M$ such that

$$
c|\rho-\tilde{\rho}| \leq|A(\rho, x)-A(\tilde{\rho}, x)| \leq C|\rho-\tilde{\rho}|, \quad \rho, \tilde{\rho}>0, \mathrm{TV} .(A(\rho, .)) \leq M(\rho), \quad x \in \mathbb{R} .
$$

Let us recall that

$$
\text { TV.f }:=\sup \left\{\sum_{j=1}^{N}\left|f\left(x_{j}\right)-f\left(x_{j-1}\right)\right|, x_{0}<x_{1}<\cdots<x_{N}\right\},
$$

where the sup is taken over all $N \geq 1$ and over all partitions $\left(x_{0}, \ldots, x_{N}\right)$.

Comments on the assumptions. Since the fluxes are one-to-one, the monotonicity assumption is stated to guarantee that the derivative of the flux $\frac{\partial A}{\partial \rho}$ does not change sign at the discontinuity points. The surjectivity of $A(., x)$ is also crucial to guarantee the existence of the adapted entropies associated with (1) and also to guarantee the existence of a solution to the Riemann problem. However, the injectivity may be relaxed, but this leads to a more complex definition of entropy solutions [7], that can be explored in a future work. Notice that the injectivity of the flux is actually implied by the bi-Lipschitz assumption ( $(\mathrm{H}-1)$. Notice that even though $A(\rho,$.$) has discontinuities, it is$ defined everywhere since it is BV, hence $(\overline{\mathrm{H}-1})$ and $(\overline{\mathrm{H}-2})$ make sense, and they still hold when $x$ is replaced by $x^{-}$or $x^{+}$. The concavity is a technical assumption that allows us to significantly reduce complexity when solving Riemann problems. It may as well be relaxed in a future work. Unlike the assumption $(\mathrm{H}-0)$ whose main purpose is to fix the ideas, the conditions $(\mathrm{H}-1)$ we impose on the regularity of the flux are crucial for existence and uniqueness. We check that the assumptions of Audusse-Perthame are satisfied, so that uniqueness of the entropy solution is guaranteed: assumption (A-1.) is direclty implied by (H-1), A-2. is obtained with $A_{-}(\rho)=c \rho$ and $A_{+}(\rho)=C \rho$ by taking $\tilde{\rho}=0$ in ( $\mathrm{H}-1)$ and using that $A(0, x)=0$ in $(\mathrm{H}-0)$, and last, $\mathrm{A}-3$. is implied by (H-1), the Lipschitz constant being $C$. We point out that in our framework, Panov solution cannot be defined. Before we go further, we mention here that even if $A$ is in a broader class than the separated variables functions, not any function $A$ is allowed. For any flux $A$, we define

$$
\operatorname{Disc}(A)=\left\{x \in \mathbb{R} \mid \exists \rho \in \mathbb{R}^{+}, A\left(\rho, x^{-}\right) \neq A\left(\rho, x^{+}\right)\right\} .
$$

Lemma 1. Consider a flux A that satisfies (H-1), then Disc $(A)$ is at most countable.

The proof of Lemma 1 is postponed to Appendix. It allows us to approximate fluxes with infinitely many discontinuities by fluxes with a finite number of discontinuities. The key technical argument to guarantee existence of the solution is the fact that

H-2 There exist a continuous function $R: \mathbb{R}^{+} \rightarrow \mathbb{R}$ and $f \in \mathrm{BV}(\mathbb{R})$ such that $|A(\rho, x)-A(\rho, y)| \leq$ $R(\rho)|f(x)-f(y)|, \quad$ for every $\rho \geq 0$, and $(x, y) \in \mathbb{R}^{2}$.

We impose $R$ to be continuous, but what matters is only the fact that it is locally bounded at any point, including $\rho=0$. Assumption $(\sqrt{\mathrm{H}-2})$ is not a consequence of $(\sqrt{\mathrm{H}-0}),(\mathrm{H}-1)$, and this leads to a deep obstacle in building the theory in the general case, as seen later 


\subsection{Existence and Non-Existence}

We identify what are the key-assumptions on the flux which are necessary to build this theory, and provide in that sense optimal general estimates on the total variation of the solution. We prove that there exists a solution that can be built via solving Riemann problems for an approximated system, and then passing the sequence of solutions to the limit via the Wave Front-Tracking method. The function we obtain at the limit coincides with the adapted entropy solution (Definition 1). This result is a generalization of [10] in the sense that we consider fluxes not included in their framework.

Theorem 1 (Existence of an entropy solution for (1)). Assume $(\mathrm{H}-0),(\mathrm{H}-1),(\mathrm{H}-2)$, and $\rho_{0} \in$ $B V(\mathbb{R})$, then there exists an adapted entropy solution $\rho \in L_{\text {loc }}^{1}\left(\mathbb{R}^{+} \times \mathbb{R}\right)$ to the Cauchy problem (1). Moreover the solution is obtained as almost everywhere limit of approximate Wave Front Tracking solutions, and it satisfies

$$
\|\rho\|_{L^{\infty}([0, T] \times \mathbb{R})} \leq \frac{C}{c}\left\|\rho_{0}\right\|_{L^{\infty}(\mathbb{R})}
$$

and

$$
T V \cdot(\rho(., t)) \leq \frac{C}{c} T V \cdot\left(\rho_{0}\right)+\frac{2}{c} \max _{\rho \in[0, M]} R(\rho) T V . f, \quad M=\frac{C}{c}\left\|\rho_{0}\right\|_{\infty},
$$

where $C$ and $c$ are the constants introduced in $(\mathrm{H}-1)$.

Next Theorem explains why the technical assumption $(\sqrt{\mathrm{H}-2})$ is crucial for a BV theory. We notice (see proof of Lemma 4 that assumptions $(\mathrm{H}-1)$ and $(\mathrm{H}-2)$ imply that

$$
\forall \rho \in \mathrm{BV}(\mathbb{R}), \mathrm{TV} . A(\rho(.), .)<+\infty .
$$

Assumption (9) on the flux is crucial, and if it is violated, there is no hope for a BV theory.

Theorem 2 (Non-Existence of a BV solution in the general case).

There exists a flux A satisfying ( $\mathrm{H}-0)$ and $(\mathrm{H}-1)$ but not $(\mathrm{H}-2)$, and an initial data $\rho_{0} \in B V(\mathbb{R})$, such that the adapted entropy solution to (1) starting from $\rho_{0}$ has an infinite total variation at any time $t>0$.

The correspondence between formulation (1) and the formulation provided by Baiti-Jenssen is not trivial for non-separated variables. In other words, for a given flux $A$, it may not be possible to find $F$ and $v$ such that (5) holds. For separated variables, the formal equivalence is clear by picking $f=v$ and $F(u, v)=a(u) v$ provided that $a \in \mathcal{C}^{2}(\mathbb{R})$, then, even in that context, our result of existence is a slight generalization to the theory developed in [10] since we allow the flux to be only Lipschitz in $\rho$, whereas their framework requires it to be at least $\mathcal{C}^{2}$. Notice that the framework considered by [10] implies that $(\mathrm{H}-2)$ is satisfied since

$$
|A(\rho, x)-A(\rho, y)|=|F(\rho, v(x))-F(\rho, v(y))| \leq\left\|F^{\prime}\right\|_{\infty}|v(x)-v(y)|,
$$

where $\left\|F^{\prime}\right\|_{\infty}$ is the Lipschitz constant of $F$, independent of $\rho$. Thus, our results generalizes [10] also for non-separated variables.

The paper is organized as follows. In Section 3, we consider a special case of (1) with piecewise constant flux $A$, which enables us to define a solution via the use of an adapted Riemann solver [21. We check that this solution is an adapted entropy solution (Definition 1). In Section 4, we derive uniform a priori estimates on the solution. Then, we approximate the general BV flux by a sequence of piecewise constant approximated fluxes and pass to the limit using the uniform BV estimates. In Section 5, we detail a counter-example preventing to generalize this theory to generic fluxes and settling the importance of assumption ( $\mathrm{H}-2)$. 


\section{The Riemann solver}

Let us define the Riemann solver we use. The initial condition is a piecewise constant function defined by

$$
\rho_{0}(x)= \begin{cases}\rho_{\ell}, & x<0, \\ \rho_{r}, & x>0,\end{cases}
$$

where $\rho_{\ell} \geq 0, \rho_{r} \geq 0$. We assume here that the discontinuities in $A(\rho,$.$) and \rho_{0}$ are localized at the same point $x=0$ since at the points where $A(\rho,$.$) is continuous, the Riemann problems are usual$ Riemann problems. The flux is written

$$
A(\rho, x)= \begin{cases}a_{-}(\rho), & x<0, \\ a_{+}(\rho), & x>0,\end{cases}
$$

where $a_{-}$and $a_{+}$are any strictly increasing and concave functions. We solve this unusual problem using a specific Riemann solver based on the theory introduced in [21] and detailed below. A Riemann solver is a function $R: \mathbb{R} \times \mathbb{R} \rightarrow \mathbb{R} \times \mathbb{R}$ which provides the left and right traces $\rho^{-}$and $\rho^{+}$ at the boundary $x=0$ of a solution to the Cauchy problem

$$
\left\{\begin{array}{cl}
\frac{\partial}{\partial t} \rho+\frac{\partial}{\partial x} a_{-}(\rho)=0, & x<0 \\
\frac{\partial}{\partial t} \rho+\frac{\partial}{\partial x} a_{+}(\rho)=0, & x>0 \\
\rho(0, x)=\rho_{0}(x),
\end{array}\right.
$$

where $\rho_{0}$ defined in $(10)$. We write $R\left(\rho_{\ell}, \rho_{r}\right)=\left(\rho^{-}, \rho^{+}\right)$. There is an equivalence between a Riemann solver $R$ and a solution to the associated Riemann problem (11).

Definition 3 (Riemann solver for discontinuous flux [21]). A function $\rho$ is said to be a weak solution to the Riemann problem (10), (11) if the traces $\left(\rho^{-}, \rho^{+}\right)$at $x=0$ are some constants that do not depend on time, and which satisfy

$R$-1. The wave $\left(\rho_{\ell}, \rho^{-}\right)$has a negative speed,

R-2. The wave $\left(\rho^{+}, \rho_{r}\right)$ has a positive speed,

R-3. The flux is preserved through the discontinuity $x=0$, i.e. $A\left(\rho^{-}, 0^{-}\right)=A\left(\rho^{+}, 0^{+}\right)$.

Proposition 1 (Existence of a bounded solution). The Riemann solver is uniquely defined by Definition 3 through

$$
R\left(\rho_{\ell}, \rho_{r}\right)=\left(\rho_{\ell}, a_{+}^{-1}\left(a_{-}\left(\rho_{\ell}\right)\right)\right) .
$$

The weak solution $\rho \in L^{\infty}([0, T] \times \mathbb{R})$ to the Riemann problem (10), (11) provided by the Riemann solver defined in Definition 3 is the unique entropy solution in the sense of Definition 1 . If moreover $a_{-}<a_{+}$, it satisfies the maximum principle

$$
\|\rho(., t)\|_{L^{\infty}(\mathbb{R})} \leq\left\|\rho_{0}\right\|_{L^{\infty}(\mathbb{R})}, \quad t \in[0, T] .
$$

For sake of clarity, we provide here the solution $\rho$ that corresponds to the Riemann solver

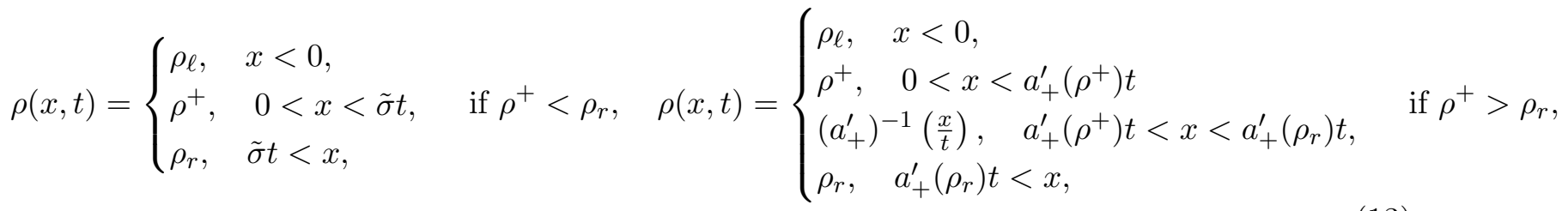



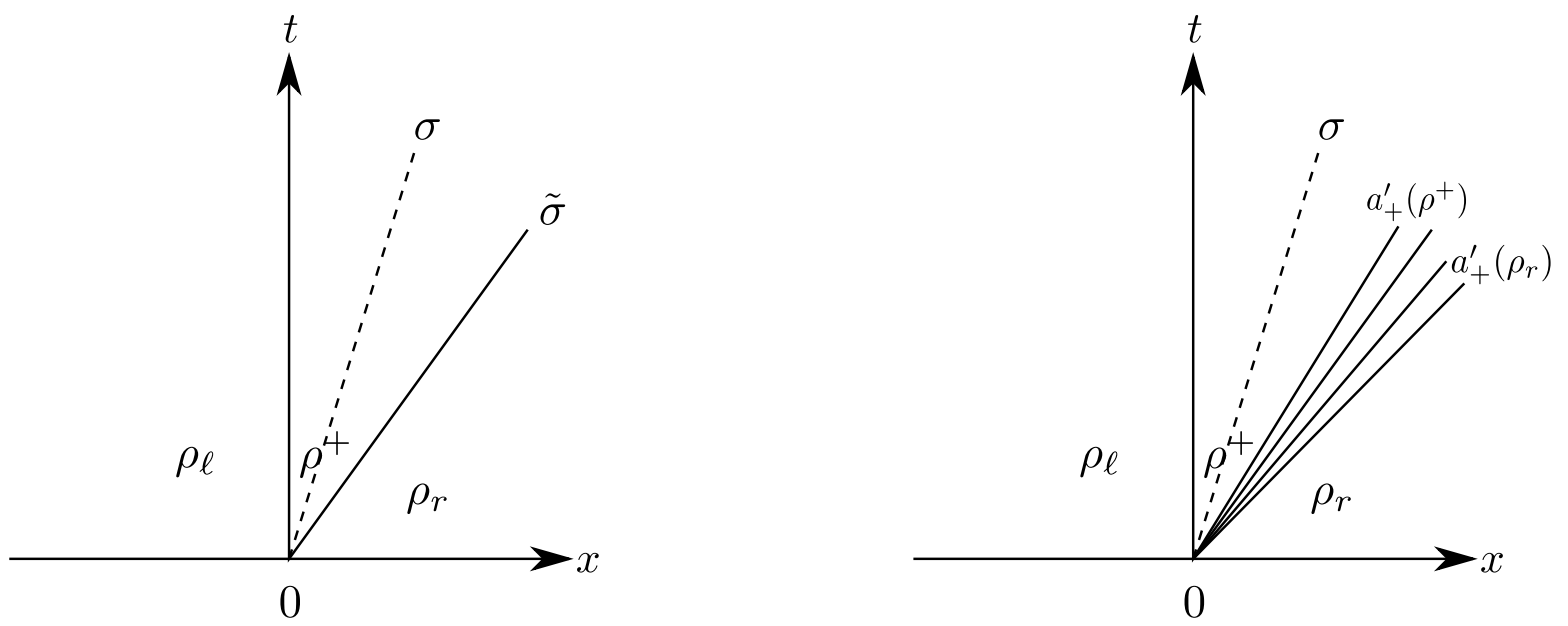

Figure 1: The solution $(13)$ is composed with two waves: a non-classical shock of velocity zero, and a shock or a rarefaction wave. The space $\mathbb{R} \times \mathbb{R}^{+}$is separated intot two domains: $\{(x, t), x<\sigma t\}$ and $\{(x, t), x>\sigma t\}$. Left: $\rho^{+}<\rho_{r}$. Right: $\rho^{+}>\rho_{r}$.

with $\tilde{\sigma}=\frac{a_{+}\left(\rho_{r}\right)-a_{+}\left(\rho^{+}\right)}{\rho_{r}-\rho^{+}}$.

Proof. Well posedness of the Riemann solver. We first prove that $\rho^{-}$is uniquely defined since that $\rho^{-}=\rho_{\ell}$. Let us first assume by contradiction that $\rho_{\ell}<\rho^{-}$. The flux $a_{-}$is concave for $x<0$ which implies that the wave $\left(\rho_{\ell}, \rho_{-}\right)$is a shock of velocity given by the Rankine-Hugoniot formula $\sigma=\left(a_{-}\left(\rho^{-}\right)-a_{-}\left(\rho_{\ell}\right)\right) /\left(\rho^{-}-\rho_{\ell}\right)$. Since $a_{-}$is increasing, the velocity $\sigma$ is positive, which contradicts $R-1$. Suppose now, still by contradiction, that $\rho_{\ell}>\rho^{-}$. The wave $\left(\rho_{\ell}, \rho_{-}\right)$is then a rarefaction wave (concavity of the flux) and the velocity of its slowest front is $a_{-}^{\prime}\left(\rho^{-}\right)>0$. This also contradicts $R-1$, and thus $\rho^{-}=\rho_{\ell}$. The trace $\rho^{+}$is uniquely determined by the relation $a_{-}\left(\rho_{\ell}\right)=a_{+}\left(\rho^{+}\right)$since $a_{+}$is one-to-one. We point out that the condition $a_{-}<a_{+}$implies that $\rho^{+} \leq \rho_{\ell}$, which guarantee that inequality $(12)$ is satisfied.

The function $(13)$ is the unique entropy solution. We prove now that the solution (13) satisfies the entropy criterion of Defintion 1, i.e. that the dissipation of entropy

$$
D(x, t)=\frac{\partial}{\partial t}\left|\rho(x, t)-k^{p}(x)\right|+\frac{\partial}{\partial x}\left\{\operatorname{sign}\left(\rho(x, t)-k^{p}(x)\right)\{A(\rho(x, t), x)-p\}\right\} \leq 0
$$

in the sense of distributions. According to formula (13), the solution (13) is composed with two waves.

The wave $\left(\rho^{+}, \rho_{r}\right)$ is entropic. The wave $\left(\rho^{+}, \rho_{r}\right)$ (shock or rarefaction wave) only propagates for $x>0$, and is entropic in the sense given by Kruzkov [25]. As a consequence, it is entropic in 
the sense of the adapted entropies, or, in other words, for $(x, t)$ such that $x>\sigma t$ with $\sigma<$ $\max \left\{\tilde{\sigma}, a_{+}^{\prime}\left(\rho^{+}\right)\right\}$and for all $p \in \mathbb{R}$,

$$
D(x, t)=\frac{\partial}{\partial t}\left|\rho(x, t)-a_{+}^{-1}(p)\right|+\frac{\partial}{\partial x}\left\{\operatorname{sign}\left(\rho(x, t)-a_{+}^{-1}(p)\right)\left\{a_{+}(\rho(x, t))-p\right\}\right\} \leq 0 .
$$

The stationary shock $\left(\rho_{\ell}, \rho^{+}\right)$is entropic. Let us now check that the shock $\left(\rho_{\ell}, \rho^{+}\right)$is entropic in the sense of the adapted entropies. The function $k^{p}$ defined by the formula (3) are expressed in that context as

$$
k^{p}(x)= \begin{cases}a_{-}^{-1}(p), & x<0 \\ a_{+}^{-1}(p), & x>0 .\end{cases}
$$

For $x<\sigma t$, the solution 130 is

$$
\rho(x, t)=\rho_{\ell}(1-H(x))+\rho^{+} H(x), \quad x<\sigma t,
$$

where $H$ is the usual Heaviside function. For $(x, t) \in \mathbb{R} \times \mathbb{R}^{+}$such that $x<\sigma t$, locally, the solution does not depend on time since it is piecewise constant with a stationary discontinuity (see Figure 1), and thus the entropy dissipation (14) is

$$
D(x, t)=\frac{\partial}{\partial x}\left[\operatorname{sign}\left(\rho_{\ell}(1-H(x))+\rho^{+} H(x)-k^{p}(x)\right)\left\{a_{-}\left(\rho_{\ell}\right)(1-H(x))+a_{+}\left(\rho^{+}\right) H(x)-p\right\}\right],
$$

which is

$$
D(x, t)=\frac{\partial}{\partial x} E(x)
$$

where

$$
\begin{aligned}
E(x) & =\operatorname{sign}\left(\rho_{\ell}(1-H(x))+\rho^{+} H(x)-k^{p}(x)\right)\left\{a_{-}\left(\rho_{\ell}\right)(1-H(x))+a_{+}\left(\rho^{+}\right) H(x)-p\right\} \\
& =\left\{\begin{array}{cc}
\operatorname{sign}\left(\rho_{\ell}-a_{-}^{-1}(p)\right)\left(a_{-}\left(\rho_{\ell}\right)-p\right), & \text { if } x<0 \\
\operatorname{sign}\left(\rho_{r}-a_{+}^{-1}(p)\right)\left(a_{+}\left(\rho_{r}\right)-p\right) & \text { if } x>0 .
\end{array}\right.
\end{aligned}
$$

Since $a_{-}$and $a_{+}$are increasing, we have

$$
E(x)= \begin{cases}\left|a_{-}\left(\rho_{\ell}\right)-p\right|, & \text { if } x<0 \\ \left|a_{+}\left(\rho^{+}\right)-p\right| & \text { if } x>0 .\end{cases}
$$

Condition $R-3$ then implies that the function $E$ is constant in a neighborhood of $x=0$, and then $D(x, t)=0$ in a neighborhood of $x=0$, which means that the entropy inequality (4) is trivially satisfied. The uniqueness of the entropy solution comes from [7]

Remark 1. The stationary shock $\left(\rho_{\ell}, \rho^{+}\right)$does not dissipate entropy. In that sense, we consider it as a non-classical shock and will distinguish it in the following from classical shocks. 


\section{Existence of a solution}

The case of general fluxes $A$ is now considered through an approximation procedure: to prove the existence of an adapted entropy solution to (1), we use the Wave-Front-Tracking algorithm 18. We approximate the initial datum $\rho_{0} \in \mathrm{BV}(\mathbb{R})$ and the flux $x \rightarrow A(\rho, x)$ by piecewise constant functions $\rho_{0, m}$ and $A_{n}(\rho,$.$) having respectively (m-1)$ and $(n-1)$ discontinuities, such that TV. $\left\{\rho_{0, m}\right\} \leq \mathrm{TV} .\left\{\rho_{0}\right\}$. We solve the Riemann problems at any discontinuity points of $\rho_{0, m}$ and $A_{n}$, using the Riemann solver defined in Section 3 . Depending whether the discontinuities of the initial profile are decreasing or increasing, they are evolving as shocks or rarefaction waves. We split rarefaction waves into rarefaction fans formed by rarefaction shocks of strength $1 / m$ (i.e. non entropic shocks) so that the profile stays piecewise constant [11. When two waves interact or a wave interacts with a discontinuity of the medium, we solve a new Riemann problem. Notice that the number of waves can only increase at $t=0$, so that the total number of waves is finite for all $n, m \geq 0$ and for every time. Thus, we can define $\rho_{n, m}$ for all $n, m$ and for every time which provides a Wave Front Tracking approximate solution (in fact it is a weak solution violating the entropy condition by a quantity going to zero with $m \rightarrow \infty$; see [11]). We first pass $m$ to the limit to obtain a solution for $\rho_{0} \in \mathrm{BV}(\mathbb{R})$ and $n$ finite. Then, passing to the limit on the sequence of approximate solution as well as on the approximated sequence of fluxes, we prove the existence of a solution to the Cauchy problem (1) for $\rho_{0} \in \mathrm{BV}(\mathbb{R})$. The key point is to obtain a uniform BV estimate for the sequence of fluxes $A_{n}\left(\rho_{n}(., t),.\right)$, where $\rho_{n}$ are Wave-Front Tracking approximations. In the general case, it is not possible to obtain BV estimates directly for $\rho_{n}$, since interactions of waves with the interface can increase the total variation of the conserved quantity [14].

Equation (1) governs the evolution of the profile made of elementary waves, and we need to understand how two elementary waves interact to estimate the time evolution of the total variation. In our context, since the flux depends on $x$, besides shock and rarefaction waves, another kind of elementary wave may be created: Non-classical shocks. We define them and describe their evolution after interaction with another classical wave.

Definition 4 (Elementary waves).

- A classical shock (CS) arising from $x=x_{d}$ is a discontinuity $\left(\rho_{\ell}, \rho_{r}\right)$ which satisfies $\rho_{\ell}<\rho_{r}$ and moves forward with velocity $s=\frac{A\left(\rho_{r}, x_{d}^{+}\right)-A\left(\rho_{\ell}, x_{d}^{+}\right)}{\rho_{r}-\rho_{\ell}}$ until it meets another elementary wave.

- A non-classical shock (NCS) is a stationary discontinuity $\left(\rho_{\ell}, \rho_{r}\right)$ at $x=x_{d}$ which satisfies for all $p \in \mathbb{R}$

$$
\frac{\partial}{\partial x}\left[\operatorname{sign}\left(G(x, t)-k^{p}(x)\right)\{A(G(x, t), x)-p\}\right]=0,
$$

where $G(x, t)=\rho_{\ell}\left(1-H\left(x-x_{d}\right)\right)+\rho_{r} H\left(x-x_{d}\right)$.

- A rarefaction shock (RS) arising from $x=x_{d}$ is a discontinuity which satisfies $\rho_{\ell}>\rho_{r}$. Its forward front moves with velocity $\frac{\partial A}{\partial \rho}\left(\rho_{r}, x_{d}^{+}\right)$and its backward front moves with velocity $\frac{\partial A}{\partial \rho}\left(\rho_{\ell}, x_{d}^{+}\right)$, until it meets another elementary wave. It is a piecewise constant approximation of a rarefaction wave.

\subsection{Piecewise constant approximations of the flux and the initial condition.}

In that part, we state and prove the existence of a solution for a piecewise constant flux $A(\rho,$.$) .$ 
Proposition 2. Under assumptions $(\mathrm{H}-0),(\mathrm{H}-1),(\mathrm{H}-2)$ for $A_{n}(\rho,$.$) uniformly piecewise constant,$ i.e. such that there exists $\left(z_{1}, \ldots z_{n}\right)$ such that $\operatorname{Disc}\left(A_{n}\right) \subset\left\{z_{1}, \ldots, z_{n}\right\}$, and for $\rho_{0, m}$ piecewise constant with discontinuities localized in $\left\{y_{j}, j=1 \cdots m\right\}$, where $\left\{z_{i}, i=1 \cdots n\right\} \cap\left\{y_{j}, j=1 \cdots m\right\}=$ $\emptyset$, we can build a solution in $L^{\infty}([0, T] \times \mathbb{R})$ to the Cauchy problem (1). In addition, the solution $\rho_{n, m}$ satisfies

$$
\left\|\rho_{n, m}\right\|_{L^{\infty}([0, T] \times \mathbb{R})} \leq \frac{C}{c}\left\|\rho_{0, m}\right\|_{L^{\infty}(\mathbb{R})}
$$

and

$$
T V \cdot\left(\rho_{n, m}(., t)\right) \leq \frac{C}{c} T V \cdot\left\{\rho_{0, m}\right\}+\frac{2}{c} \max _{[0, M]} R(.) \quad T V \cdot\{f\}, \quad M=\frac{C}{c}\left\|\rho_{0, m}\right\|_{\infty} .
$$

The rest of this section is devoted to the proof of Proposition 2. To simplify notations, we drop the $m$ - and $n$-dependence in the notation for $\rho_{n, m}, A_{n}$ and $\rho_{0, m}$ and write $\rho, A$ and $\rho_{0}$. We prove in this part that the total variation of the solution is bounded independently of $m$ and $n$. Since the approximation $\rho$ is piecewise constant, the total variation of $\rho(., t)$ is defined as

$$
\text { TV. } \rho(., t)=\sum_{i}\left|\rho\left(x_{i}^{+}, t\right)-\rho\left(x_{i}^{-}, t\right)\right|, \quad x_{i} \text { are the discontinuity points of } \rho .
$$

The points of discontinuity $x_{i}$ actually depend on $t$, but we drop this dependence for simplifying the notations. We distinguish the discontinuities of $\rho$ as follows

$$
\operatorname{TV} \cdot(\rho(., t))=\operatorname{TV}_{C} \cdot(\rho(., t))+\operatorname{TV}_{N C} \cdot(\rho(., t)),
$$

where

Definition 5 (Total variation of non-classical and classical shocks). We define for all time $t>0$, except for the times at which two waves interact:

$$
\begin{gathered}
T V_{C} \cdot(\rho(., t)):=\sum_{j=1}^{I}\left|\rho\left(x_{j}^{+}, t\right)-\rho\left(x_{j}^{-}, t\right)\right|,\left(x_{1}, \ldots, x_{I}\right) \in \mathbb{R}^{I} \mid x_{j} \notin\left\{z_{i}, i=1 \ldots n\right\}, j=1 \ldots I, \\
T V_{N C} \cdot(\rho(., t)):=\sum_{i=1}^{n}\left|\rho\left(z_{i}^{+}, t\right)-\rho\left(z_{i}^{-}, t\right)\right| .
\end{gathered}
$$

The total variation TV. $\rho(., t)$ evolves with time 1) when two waves interact, and 2) at time $t=0$ because of the discontinuous medium. Two waves interact if they reach the same location at the same time. There are three types of waves: classsical shocks, rarefaction shocks and non-clasical shocks. Non classical shocks are stationary, so that two non classical shocks can never interact. Since the flux $A(., x)$ is strictly increasing, waves propagate from left to the right, thus the only way a classical wave meets a non classical shock is if the classical wave arrives from the left. Last, two classical waves can interact, as in usual conservation laws". To summarize, the interactions waves that can occur are of 3 types:

1. When a classical shock arrives at $x=z_{i}$, we have an interaction of type CS/NCS, and this gives rise to a classical shock and a non-classical shock.

2. When a rarefaction shock arrives at $x=z_{i}$, we have an interaction of type RS/NCS, and this gives rise to another rarefaction shock and a non-classical shock.

3. Two waves of type CS/RS, CS/CS, RS/CS can interact and it gives rise to one wave of type CS or RS (usual Riemann problem). 

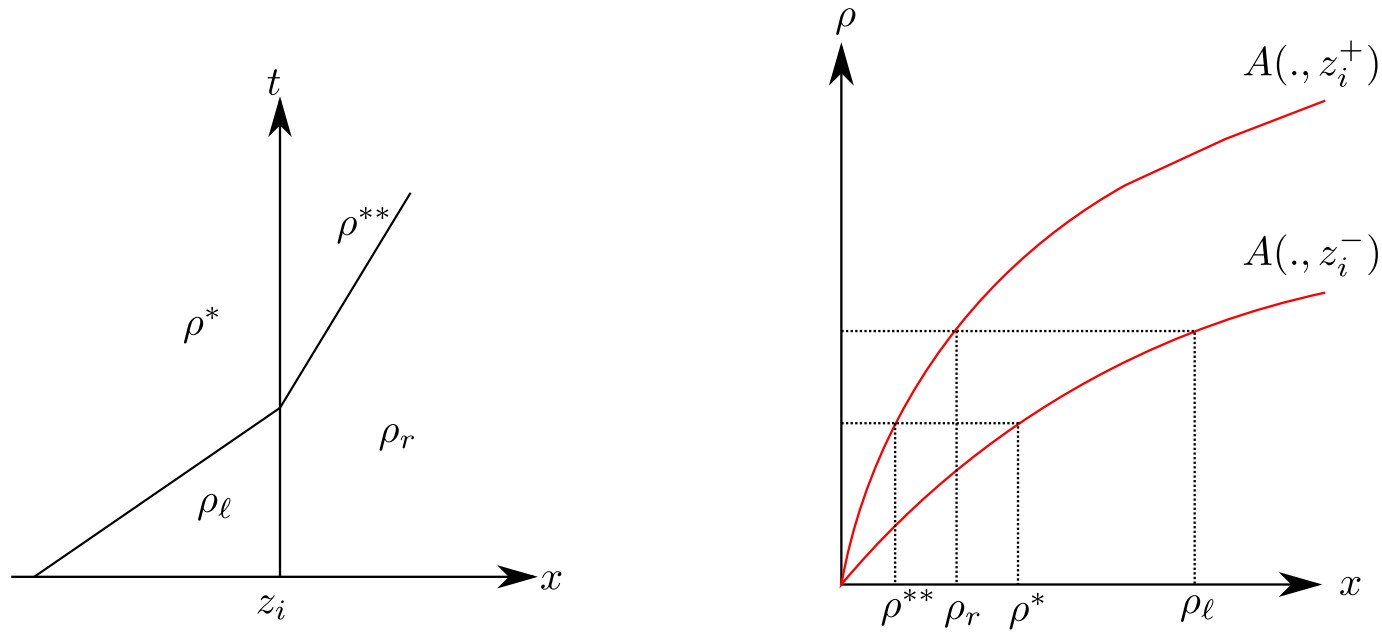

Figure 2: Waves interaction. A wave $\left(\rho^{*}, \rho_{\ell}\right)$ meets a non-classical shock $\left(\rho_{\ell}, \rho_{r}\right)$ This gives rise to a new state $\rho^{* *}$ (Left) obtained by the Rankine Hugoniot conditions (Right).

See Figure 2 for an illustration. We now describe the waves creations at time $t=0$. Let us remind that $\rho_{0}$ is continuous at the points where the medium is discontinuous, so that $\rho_{0}\left(z_{i}\right)$ is well-defined. At time $t=0$,

1. At the points where $\rho_{0}$ is discontinuous, a classical wave is created. It may be a classical shock or a rarefaction shock depending on whether $\rho_{0}$ is increasing or decreasing.

2. At the points $z_{i}$ of discontinuity of $A(\rho,$.$) , we have the creation of a classical wave \left(\rho_{i}^{*}, \rho_{0}\left(z_{i}\right)\right)$ and creation of non classical shock $\left(\rho_{0}\left(z_{i}\right), \rho_{i}^{*}\right)$.

See Figure 3 for an illustration. At time $t=0$, the discontinuities in $\rho_{0}$ can neither be classified as classical nor non classical shocks so that we do not distinguish $\operatorname{TV}_{C} \cdot\left\{\rho_{0}\right\}$ and $\operatorname{TV}_{N C} \cdot\left\{\rho_{0}\right\}$, but we merely have

$$
\mathrm{TV} \cdot\left\{\rho_{0}\right\}=\sum_{j=1}^{m}\left|\rho_{0}\left(y_{j}^{+}\right)-\rho_{0}\left(y_{j}^{-}\right)\right| .
$$

The sequence of the following lemmas ends in the proof of Proposition 2 .

Lemma 2 (TV. bounds on the fluxes). We denote by $\rho$ the approximated solution to (1), where the flux $A$ is piecewise constant. Then, for almost every time (except interaction times), we have

$$
\begin{aligned}
& T V_{C} \cdot\left(A(\rho(., t), .) \leq T V \cdot\left(A\left(\rho_{0}(.), .\right),\right.\right. \\
& T V_{N C} \cdot(A(\rho(., t), .)=0 .
\end{aligned}
$$



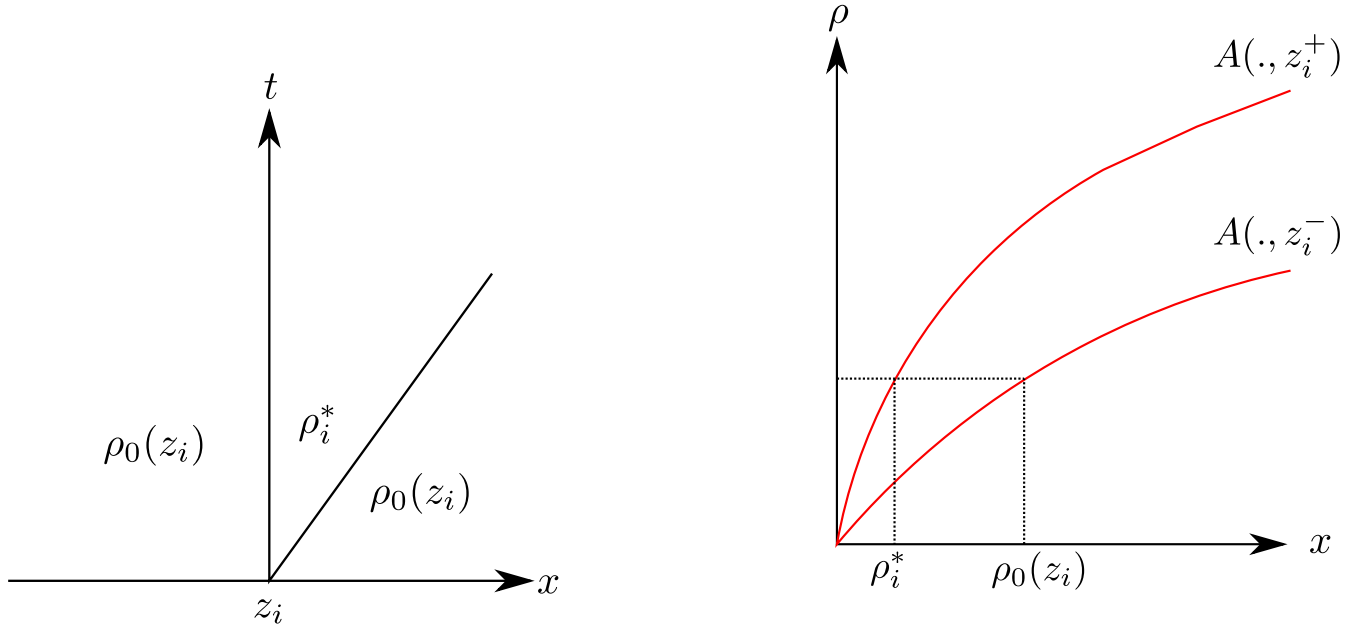

Figure 3: Wave creation. Two waves $\left(\rho_{0}\left(z_{i}\right), \rho_{i}^{*}\right)$ and $\left(\rho_{i}^{*}, \rho_{0}\left(z_{i}\right)\right)$ emerge at time $t=0$. The new state $\rho_{i}^{*}$ (Left) is obtained by the Rankine Hugoniot conditions (Right).

Proof. We have

$$
\begin{array}{r}
\operatorname{TV}_{C} \cdot(A(\rho(., t), .))=\sum_{j=1}^{I}\left|A\left(\rho\left(x_{j}, t\right), x_{j}\right)-A\left(\rho\left(x_{j-1}, t\right), x_{j-1}\right)\right|,\left(x_{1}, \ldots, x_{I}\right) \in \mathbb{R}^{I} \mid x_{j} \neq z_{i}, \\
j=1 \ldots I, i=1 \ldots n
\end{array}
$$

The total variation of $\rho(., t)$ can only decrease when two classical waves interact ([18], Chapter $\mathrm{XIV})$, and thus, $\mathrm{TV}_{C} \cdot A(\rho(., t),$.$) can only decrease as well since A(., x)$ is increasing and since only classical waves can interact at $x \neq z_{i}$. Let us assume that a classical wave interacts with a non classical shock at time $t$ and at $x=z_{i}$, then we have (see Figure 2 )

$\operatorname{TV}_{C} \cdot\left(A\left(\rho\left(., t^{+}\right),.\right)\right)-\operatorname{TV}_{C} \cdot\left(A\left(\rho\left(., t^{-}\right),.\right)\right)=\left(\left|A\left(\rho^{* *}, z_{i}^{+}\right)-A\left(\rho_{r}, z_{i}^{+}\right)\right|\right)-\left(\left|A\left(\rho^{*}, z_{i}^{-}\right)-A\left(\rho_{\ell}, z_{i}^{-}\right)\right|\right)$,

where the Rankine-Hugoniot conditions impose that

$$
\begin{aligned}
& A\left(\rho^{*}, z_{i}^{-}\right)=A\left(\rho^{* *}, z_{i}^{+}\right) \\
& A\left(\rho_{\ell}, z_{i}^{-}\right)=A\left(\rho_{r}, z_{i}^{+}\right) .
\end{aligned}
$$

Hence, we have

$$
\operatorname{TV}_{C} \cdot\left(A\left(\rho\left(., t^{-}\right), .\right)\right)=\operatorname{TV}_{C} \cdot\left(A\left(\rho\left(., t^{+}\right), .\right)\right),
$$

which implies that the total variation of the flux does not evolve after an interaction NCS-CS or NCS-RS, i.e.

$$
\operatorname{TV}_{C} \cdot(A(\rho(., t), .))=\mathrm{TV}_{C} \cdot\left(A\left(\rho\left(., 0^{+}\right), .\right)\right), \quad t>0 .
$$

Let us now explore how the total variation is modified initially. At time $t=0, n$ classical waves $\left(\rho_{i}^{*}, \rho_{0}\left(x_{i}\right)\right)$ are simultaneously created from the $n$ discontinuities of the medium, and the new states 
$\rho_{i}^{*}$ satisfy $A\left(\rho_{0}\left(z_{i}\right), z_{i}^{-}\right)=A\left(\rho_{i}^{*}, z_{i}^{+}\right)$. At time $t=0$, we have

$$
\left.\operatorname{TV} .\left(A\left(\rho_{0}(.), .\right)\right)=\sum_{i=1}^{n} \mid A\left(\rho_{0}\left(z_{i}\right)\right), z_{i}^{+}\right)-A\left(\rho_{0}\left(z_{i}\right), z_{i}^{-}\right)\left|+\sum_{j=1}^{m}\right| A\left(\rho_{0}\left(y_{j}^{-}\right), y_{j}\right)-A\left(\rho_{0}\left(y_{j}^{+}\right), y_{j}\right) \mid .
$$

At time $t=0^{+}$, we have $n+m$ classical waves and $n$ non-classical shocks

$$
\begin{aligned}
\operatorname{TV}_{C} \cdot\left(A\left(\rho\left(., 0^{+}\right), .\right)\right) & =\sum_{i=1}^{n}\left|A\left(\rho_{i}^{*}, z_{i}^{+}\right)-A\left(\rho_{0}\left(z_{i}\right), z_{i}^{+}\right)\right|+\sum_{j=1}^{m}\left|A\left(\rho_{0}\left(y_{j}^{-}\right), y_{j}\right)-A\left(\rho_{0}\left(y_{j}^{+}\right), y_{j}\right)\right| \\
& =\operatorname{TV} \cdot\left(A\left(\rho_{0}(.), .\right)\right),
\end{aligned}
$$

and the first statement of Lemma 2 is proved combining $(15)$ and $(16)$. The result for non classical shocks holds because of the flux conservation through the discontinuities $z_{i}$.

In the following lemma, we state that the continuity of the flux implies that the solution stays bounded for all time

Lemma 3 ( $L^{\infty}$ bound). We denote by $\rho$ the approximated solution to (1), where the flux $A$ is piecewise constant. Then

$$
\|\rho(., t)\|_{L^{\infty}(\mathbb{R})} \leq \frac{C}{c}\left\|\rho_{0}\right\|_{L^{\infty}(\mathbb{R})} .
$$

Proof. We have, using (

$$
\|\rho(., t)\|_{L^{\infty}(\mathbb{R})} \leq \frac{1}{c}\|A(\rho(., t), .)\|_{L^{\infty}(\mathbb{R})} .
$$

The flux conservation through both classical and non-classical waves implies

$$
\|A(\rho(., t), .)\|_{L^{\infty}(\mathbb{R})}=\left\|A\left(\rho_{0}(.), .\right)\right\|_{L^{\infty}(\mathbb{R})} .
$$

Then

$$
\|\rho(., t)\|_{L^{\infty}(\mathbb{R})} \leq \frac{1}{c}\left\|A\left(\rho_{0}(.), .\right)\right\|_{L^{\infty}(\mathbb{R})} \leq \frac{C}{c}\left\|\rho_{0}\right\|_{L^{\infty}(\mathbb{R})} .
$$

Notice that this is not a maximum principle since it may happen that $C>c$.

Lemma 4 (TV. bounds for $\rho_{n}$ ). We denote by $\rho$ the approximated solution to (1), where the flux $A$ is piecewise constant. Then,

$$
\begin{gathered}
T V_{C} \cdot(\rho(., t)) \leq \frac{1}{c}\left(\max _{\left[0,\left\|\rho_{0}\right\|_{\infty}\right]} R(.) T V .\{f\}+C T V \cdot\left\{\rho_{0}\right\}\right), \\
T V_{N C \cdot(\rho(., t))} \leq \frac{1}{c} \max _{[0, M]} R(.) T V .\{f\}, \quad \text { where } M=\frac{C}{c}\left\|\rho_{0}\right\|_{\infty} .
\end{gathered}
$$

Proof. The solution $\rho$ is piecewise constant, so that its total variation is uniquely determined by its discontinuities.

First Step : $\mathbf{T V}_{C} \cdot(\rho(., t))$. At time $t$, the number of classical waves is at most $N+J$. Let us denote by $\left(\rho_{j}^{-}, \rho_{j}^{+}\right)$the wave number $j$ localized at $x=x_{j}$. The localization $x_{j}$ does not coincide 
with a discontinuity point $z_{i}$ (except maybe for a finite number of times we do not pick here). We first control the total variation of $\rho(., t)$ by the total variation of $A(\rho(., t),$.

$$
\operatorname{TV}_{C} \cdot(\rho(., t))=\sum_{j=1}^{n+m}\left|\rho_{j}^{+}-\rho_{j}^{-}\right| \leq \frac{1}{c} \sum_{j=1}^{n+m}\left|A\left(\rho_{j}^{+}, x_{j}\right)-A\left(\rho_{j}^{-}, x_{j}\right)\right| \leq \frac{1}{c} \operatorname{TV} \cdot(A(\rho(., t), .)) .
$$

We now use Lemma 2 to obtain

$$
\mathrm{TV}_{C} \cdot(\rho(., t)) \leq \frac{1}{c} \mathrm{TV} \cdot\left(A\left(\rho_{0}(.), .\right)\right) .
$$

For all $(K+1)$-uplets $x_{0}<x_{1}<\cdots<x_{K}$, for all $\rho \in \mathrm{BV}(\mathbb{R})$, using $(\mathrm{H}-1)$ and $(\mathrm{H}-2)$,

$$
\begin{aligned}
\sum_{j=1}^{K}\left|A\left(\rho\left(x_{j}\right), x_{j}\right)-A\left(\rho\left(x_{j-1}\right), x_{j-1}\right)\right| & \leq \sum_{j=1}^{K}\left|A\left(\rho\left(x_{j}\right), x_{j}\right)-A\left(\rho\left(x_{j}\right), x_{j-1}\right)\right| \\
& +\sum_{j=1}^{K}\left|A\left(\rho\left(x_{j}\right), x_{j-1}\right)-A\left(\rho\left(x_{j-1}\right), x_{j-1}\right)\right| \\
\leq & \sum_{j=1}^{K} R\left(\rho\left(x_{j}\right)\right)\left|f\left(x_{j}\right)-f\left(x_{j-1}\right)\right|+\sum_{j=1}^{K} C\left|\rho\left(x_{j}\right)-\rho\left(x_{j-1}\right)\right| .
\end{aligned}
$$

Hence,

$$
\operatorname{TV} \cdot\left(A\left(\rho_{0}(.), .\right)\right) \leq \max _{\left[0,\left\|\rho_{0}\right\|_{\infty}\right]} R(.) \operatorname{TV} \cdot\{f\}+C \operatorname{TV} \cdot\left\{\rho_{0}\right\} .
$$

The combination of (17) and (18) gives us the first line of Lemma 4 .

Second Step : $\mathbf{T V}_{N C} \cdot(\rho(., t))$. The non classical shocks are the waves $\left(\rho\left(z_{i}^{-}, t\right), \rho\left(z_{i}^{+}, t\right)\right)$ such that

$$
A\left(\rho\left(z_{i}^{-}, t\right), z_{i}^{-}\right)=A\left(\rho\left(z_{i}^{+}, t\right), z_{i}^{+}\right) .
$$

Thus, we have, using (H-1)

$$
\operatorname{TV}_{N C \cdot}\{\rho(., t)\}=\sum_{i=1}^{n}\left|\rho\left(z_{i}^{+}, t\right)-\rho\left(z_{i}^{-}, t\right)\right| \leq \frac{1}{c} \sum_{i=1}^{n}\left|A\left(\rho\left(z_{i}^{+}, t\right), z_{i}^{+}\right)-A\left(\rho\left(z_{i}^{-}, t\right), z_{i}^{+}\right)\right|
$$

and then by 19

$$
\operatorname{TV}_{N C} \cdot\{\rho(., t)\} \leq \frac{1}{c} \sum_{i=1}^{n}\left|A\left(\rho\left(z_{i}^{-}, t\right), z_{i}^{-}\right)-A\left(\rho\left(z_{i}^{-}, t\right), z_{i}^{+}\right)\right|,
$$

which is, using $(\mathrm{H}-2)$ and Lemma 3

$$
\operatorname{TV}_{N C} \cdot\{\rho(., t)\} \leq \frac{1}{c} \sum_{i=1}^{n} R\left(\rho\left(z_{i}^{-}, t\right)\right)\left|f\left(z_{i}^{-}\right)-f\left(z_{i}^{+}\right)\right|
$$

which end the proof of Lemma 4, since $M \geq\left\|\rho_{0}\right\|_{\infty}$. That ends the proof of Proposition 2 


\subsection{Infinite number of discontinuities}

We describe here how we pass to the limit and ends the proof of Theorem 1 .

Lemma 5. Under assumptions ( $\mathrm{H}-0),(\mathrm{H}-1),(\mathrm{H}-2)$ for $A(\rho,$.$) uniformly piecewise constant, i.e.$ such that there exists $\left(z_{1}, \ldots z_{n}\right)$ such that $\operatorname{Disc}(A) \subset\left\{z_{1}, \ldots, z_{n}\right\} . \rho_{0} \in B V(\mathbb{R})$ we can build a solution in $L^{\infty}([0, T] \times \mathbb{R})$ to the Cauchy problem (1). In addition, the solution $\rho$ satisfies

$$
\|\rho\|_{L^{\infty}([0, T] \times \mathbb{R})} \leq \frac{C}{c}\left\|\rho_{0}\right\|_{L^{\infty}(\mathbb{R})}
$$

and

$$
T V \cdot(\rho(., t)) \leq \frac{C}{c} T V \cdot\left\{\rho_{0}\right\}+\frac{2}{c} \max _{[0, M]} R(.) \quad T V \cdot\{f\}, \quad M=\frac{C}{c}\left\|\rho_{0}\right\|_{\infty} .
$$

Proof. Lemma 5 is obtained by approximating $\rho_{0}$ by piecewise a constant function $\rho_{0, m}$ such that TV. $\left\{\rho_{0, m}\right\} \leq \mathrm{TV} .\left\{\rho_{0}\right\}$, and by letting $m \rightarrow \infty$ (classical Wave Front Tracking algorithm) in Proposition 2 .

Lemma 6. Given a flux $A$ which satisfies $(\mathrm{H}-0),(\sqrt{\mathrm{H}-1}),(\mathrm{H}-2)$, we can build a sequence of piecewise constant fluxes $A_{n}$ (with $(n-1)$ discontinuities) satisfying ( $\left.\mathrm{H}-0\right),(\mathrm{H}-1),(\mathrm{H}-2)$ as well and such that

1. the fluxes $A_{n}$ approximate the flux $A$ in the sense that

$$
\forall \rho \in \mathbb{R}, \quad\left\|A_{n}(\rho, .)-A(\rho, .)\right\|_{L^{\infty}(\mathbb{R})} \leq \varepsilon(n) R(\rho), \quad \varepsilon(n) \rightarrow 0 \text { as } n \rightarrow \infty .
$$

2. If we denote by $k_{n}^{p}$ and $k^{p}$ the functions which respectively satisfy $A_{n}\left(k_{n}^{p}(x), x\right)=p$ and $A\left(k^{p}(x), x\right)=p$, then

$$
\lim _{n \rightarrow \infty}\left\|k_{n}^{p}-k^{p}\right\|_{L^{1}(\mathbb{R})}=0 .
$$

Proof. First Step. Constructing the $A_{n}$. The construction of the piecewise approximation of the flux $A$ is based on the constructive proof of Lemma 2.2, [11, which states that any BV function can be approximated in $L^{\infty}$ by a sequence of piecewise constant functions. We adapt the proof in our case, basically by showing that we can perform the same construction independently of $\rho$. Let us define the right-continuous function

$$
F(x)=\mathrm{TV} .\left\{f_{\mid(-\infty, x]}\right\}=\sup \left\{\sum_{j=1}^{N}\left|f\left(x_{j}\right)-f\left(x_{j-1}\right)\right| ; N \geq 1, x_{0}<x_{1}<\cdots<x_{N}=x\right\}
$$

where $f$ is defined in $(\mathrm{H}-2)$ and assumed to be right-continuous (with no restriction). Given $\varepsilon>0$, let $n$ be the largest integer such that $n \varepsilon \leq \mathrm{TV} .\{f\}$, and consider the points

$$
z_{0}=-\infty, \quad z_{n}=+\infty, \quad z_{j}=\min \{x ; F(x) \geq j \varepsilon\}, j=1 \ldots n-1 .
$$

Defining

$$
f_{n}(x)=f\left(z_{j}\right), \quad A_{n}(\rho, x)=A\left(\rho, z_{j}\right), \quad \text { for } x \in\left[z_{j}, z_{j+1}\right),
$$

we guarantee that the piecewise constant flux $A_{n}$ satisfies $(\mathrm{H}-0)$ and $(\mathrm{H}-1)$ with the same constants as $A$, and $(\mathrm{H}-2)$ is satisfied as well since there exists $i, j \in[0, N]$ such that

$$
\left|A_{n}(\rho, x)-A_{n}(\rho, y)\right|=\left|A\left(\rho, z_{i}\right)-A\left(\rho, z_{j}\right)\right| \leq R(\rho)\left|f\left(z_{i}\right)-f\left(z_{j}\right)\right|=R(\rho)\left|f_{n}(x)-f_{n}(y)\right|,
$$


and

$$
\mathrm{TV} .\left\{f_{n}\right\} \leq \mathrm{TV} \cdot\{f\} .
$$

Notice that being of bounded variations, $f$ and $A(\rho,$.$) both admit a finite limit at -\infty$. Finally, the estimate 20 is guaranteed since for $x \in\left[z_{j}, z_{j+1}\right)$,

$$
\left|A(\rho, x)-A_{n}(\rho, x)\right|=\left|A(\rho, x)-A\left(\rho, z_{j}\right)\right| \leq R(\rho)\left|f\left(z_{j}\right)-f(x)\right| \leq R(\rho) \varepsilon(n) .
$$

Second Step. Convergence of the $\left(k_{n}^{p}\right)_{n}$. We first claim that the $k_{n}^{p}$ are uniformly bounded in $L^{\infty}(\mathbb{R})$. Indeed, we have, using ( $\left.\mathrm{H}-1\right)$

$$
\left|k_{n}^{p}(x)\right| \leq \frac{1}{c}\left|A_{n}\left(k_{n}^{p}(x), x\right)\right|=\frac{|p|}{c}, \quad x \in \mathbb{R} .
$$

Secondly, we claim that the $k_{n}^{p}$ are uniformly bounded in $\mathrm{BV}(\mathbb{R})$. Indeed, the functions $k_{n}^{p}$ are piecewise constant and we have

$$
\mathrm{TV} .\left\{k_{n}^{p}\right\}=\sum_{i=1}^{n}\left|k_{n}^{p}\left(z_{i}^{+}\right)-k_{n}^{p}\left(z_{i}^{-}\right)\right| \leq \frac{1}{c} \sum_{i=1}^{n}\left|A\left(k_{n}^{p}\left(z_{i}^{+}\right), z_{i}^{+}\right)-A\left(k_{n}^{p}\left(z_{i}^{-}\right), z_{i}^{+}\right)\right| .
$$

Since $A\left(k_{n}^{p}\left(z_{i}^{+}\right), z_{i}^{+}\right)=p=A\left(k_{n}^{p}\left(z_{i}^{-}\right), z_{i}^{-}\right)$, we obtain

$$
\begin{aligned}
\mathrm{TV} .\left\{k_{n}^{p}\right\} \leq \frac{1}{c} \sum_{i=1}^{n}\left|A\left(k_{n}^{p}\left(z_{i}^{-}\right), z_{i}^{-}\right)-A\left(k_{n}^{p}\left(z_{i}^{-}\right), z_{i}^{+}\right)\right| & \leq \frac{1}{c} \sum_{i=1}^{n} R\left(k_{n}^{p}\left(z_{i}^{-}\right)\right)\left|f_{n}\left(z_{i}^{-}\right)-f_{n}\left(z_{i}^{+}\right)\right| \\
& \leq \frac{1}{c} \max _{\rho \in[0,|p| / c]} R(\rho) \mathrm{TV} .\{f\} .
\end{aligned}
$$

Helly theorem assures that there exists $k^{p} \in L_{l o c}^{1}(\mathbb{R})$ such that $\left\|k_{n}^{p}-k^{p}\right\|_{L^{1}(\mathbb{R})} \underset{n \rightarrow \infty}{\longrightarrow}$. Let us now check that $A\left(k^{p}(x), x\right)=p$. We have

$$
\begin{aligned}
\left|A\left(k^{p}(x), x\right)-p\right| & =\left|A\left(k^{p}(x), x\right)-A_{n}\left(k_{n}^{p}(x), x\right)\right| \\
& \leq\left|A\left(k^{p}(x), x\right)-A_{n}\left(k^{p}(x), x\right)\right|+\left|A_{n}\left(k^{p}(x), x\right)-A_{n}\left(k_{n}^{p}(x), x\right)\right| .
\end{aligned}
$$

According to 201 ,

$$
\left|A\left(k^{p}(x), x\right)-A_{n}\left(k^{p}(x), x\right)\right| \leq \max _{\rho \in[0,|p| / c]} R(\rho) \varepsilon(n),
$$

and using (21)

$$
\left|A_{n}\left(k^{p}(x), x\right)-A_{n}\left(k_{n}^{p}(x), x\right)\right| \leq C\left|k^{p}(x)-k_{n}^{p}(x)\right| \underset{n \rightarrow \infty}{\rightarrow} 0, \quad \text { a.e. }
$$

which ends the proof of Lemma 6 .

Proof of Theorem (1). We approximate the flux $x \rightarrow A(\rho, x)$ by $A_{n}(\rho,$.$) given by Lemma 6$. According to Lemma for all $n \geq 0$, there exists $\rho_{n} \in L^{\infty}([0, T] \times \mathbb{R})$ uniformly bounded in BV and such that we have in the distributional sense

$$
\partial_{t}\left|\rho_{n}(x, t)-k_{n}^{p}(x)\right|+\operatorname{sign}\left(\rho_{n}(x, t)-k_{n}^{p}(x)\right)\left\{A_{n}\left(\rho_{n}(x, t), x\right)-A_{n}\left(k_{n}^{p}(x), x\right)\right\} \leq 0 .
$$

Following [1] Chapter VI page 113, the $\rho_{n}$ are uniformly Lipschitz in time with values in $L^{1}(\mathbb{R})$. Then, Lemma 5 combined with Theorem 2.6 of [11] gives us that $\rho_{n}$ is uniformly bounded in 
$\mathrm{BV}([0, T] \times \mathbb{R})$ and thus there exists $\rho \in L_{\text {loc }}^{1}([0, T) \times \mathbb{R})$ such that after extraction of a subsequence, $\left\|\rho_{n}-\rho\right\|_{L^{1}([0, T] \times \mathbb{R})} \rightarrow 0$ as $n \rightarrow \infty$. We combine this with [21), and

$$
\begin{aligned}
\left|A_{n}\left(\rho_{n}(x, t), x\right)-A(\rho(x, t), x)\right| & \leq\left|A_{n}\left(\rho_{n}(x, t), x\right)-A_{n}(\rho(x, t), x)\right|+\left|A_{n}(\rho(x, t), x)-A(\rho(x, t), x)\right| \\
& \leq C\left|\rho_{n}(x, t)-\rho(x, t)\right|+\varepsilon(n) R(\rho(x, t)),
\end{aligned}
$$

so that $A_{n}\left(\rho_{n}(x, t), x\right)$ converges strongly toward $A(\rho(x, t), x)$, after extracting a subsequence. We can now pass to the limit in the entropy inequality $(22)$, and we get that $\rho$ satisfies

$$
\partial_{t}\left|\rho(x, t)-k^{p}(x)\right|+\partial_{x}\left(\operatorname{sign}\left(\rho(x, t)-k^{p}(x)\right)\left\{A(\rho(x, t), x)-A\left(k^{p}(x), x\right)\right\}\right) \leq 0, \quad \mathcal{D}^{\prime}(\mathbb{R}),
$$

which means that $\rho$ is the unique entropy solution of (1).

\section{Non-Existence in the general case : A counter example.}

This section is devoted to the proof of Theorem 2. We show that if $(9)$ is violated, discontinuities in $x$ may immediately generate a blow up: even if the initial condition has a finite total variation at time $t=0$, the total variation can become infinite at time $t=0^{+}$. We then exhibit a flux $A$ satisfying $(\mathrm{H}-0)$ and $(\mathrm{H}-1)$, and an initial datum $\rho_{0} \in \mathrm{BV}(\mathbb{R})$ such that

$$
\sum_{n=1}^{+\infty}\left|A\left(\rho_{0}\left(n^{+}\right), n^{+}\right)-A\left(\rho_{0}\left(n^{-}\right), n^{-}\right)\right|=+\infty .
$$

Our proof is divided in two steps:

- Step 1: Assuming that there exists a continuous initial datum $\rho_{0} \in \mathrm{BV}(\mathbb{R})$ and a flux $A$ satisfying $(\mathrm{H}-0),(\mathrm{H}-1)$ such that the key point $(9)$ is violated, prove that the total variation of the solution $\rho\left(., 0^{+}\right)$is unbounded.

- Step 2: Prove that there exists a continuous function $\rho_{0} \in \mathrm{BV}(\mathbb{R})$ and a flux $A$ satisfying (H-0), (H-1) such that the key point (9) is violated.

\subsection{Step 1. A necessary condition to prevent blow up at time $t=0^{+}$.}

In this subsection, we focus on Step 1. We assume that the pair $\left(A, \rho_{0}\right)$ satisfies $(23)$. Since $\rho_{0}$ is continuous, we have

$$
\begin{aligned}
\operatorname{TV} .\left\{\rho\left(., 0^{+}\right)\right\} \geq \sum_{n=1}^{+\infty}\left|\rho\left(n^{+}, 0^{+}\right)-\rho\left(n^{-}, 0^{+}\right)\right| & \geq \frac{1}{C} \sum_{n=1}^{+\infty}\left|A\left(\rho\left(n^{+}, 0^{+}\right), n^{+}\right)-A\left(\rho\left(n^{-}, 0^{+}\right), n^{+}\right)\right| \\
& =\frac{1}{C} \sum_{n=1}^{+\infty}\left|A\left(\rho_{0}(n), n^{-}\right)-A\left(\rho_{0}(n), n^{+}\right)\right|,
\end{aligned}
$$

since $A\left(\rho\left(n^{+}, 0^{+}\right), n^{+}\right)=A\left(\rho\left(n^{-}, 0^{+}\right), n^{-}\right)=A\left(\rho_{0}(n), n^{-}\right)$(See Figure 2 ) Notice that time continuity (see Definition 1) of the solution is needed here. Then, the total variation of $\rho\left(., 0^{+}\right)$is unbounded. 


\subsection{Step 2. Constructing $A$ and $\rho_{0}$.}

We exhibit in this part a flux $A$ such that $\operatorname{Disc}(A) \subset \mathbb{N}$, where $\mathbb{N}$ denotes here the set of strictly positive integers, and an initial condition $\rho_{0} \in \mathrm{BV}([1,+\infty))$ such that $(9)$ is violated. Let us first consider the bounded initial condition

$$
\rho_{0}(x)=\frac{1}{x}, \quad x \in[1,+\infty), \quad \rho_{0}(x)=1, \quad x<1,
$$

which satisfies TV. $\left\{\rho_{0}\right\}<\infty$. We then consider the flux $A(\rho, x)$ defined as, for all $n$ even

$$
A(\rho, x)= \begin{cases}a_{n}(\rho), & n \leq x<n+1 \\ \bar{a}_{n}(\rho), & n-1 \leq x<n\end{cases}
$$

where $a_{n}$ and $\bar{a}_{n}$ are defined as

$$
a_{n}(\rho)=\left\{\begin{array}{l}
2 \rho, \quad \rho \in\left(0, \frac{1}{2 n}\right), \\
\frac{2}{3}\left(\rho+\frac{1}{n}\right), \quad \rho \in\left(\frac{1}{2 n}, \infty\right),
\end{array} \quad \bar{a}_{n}(\rho)=\left\{\begin{array}{l}
\rho, \quad \rho \in\left(0, \frac{2}{n}\right), \\
\frac{2}{3}\left(\rho+\frac{1}{n}\right), \quad \rho \in\left(\frac{2}{n}, \infty\right) .
\end{array}\right.\right.
$$

(see Figure 4, Left). For $x<0$, we assume $A(\rho, x)=A(\rho, 0)$.

1. The flux $A$ satisfies the requirements $(\mathrm{H}-0)$, (H-1). For all $(\rho, x) \in \mathbb{R}^{2}, \rho \rightarrow A(\rho, x)$ is strictly increasing and concave, and uniformly Lipschitz in the sense that

$$
\frac{2}{3}|\rho-\tilde{\rho}| \leq|A(\rho, x)-A(\tilde{\rho}, x)| \leq 2|\rho-\tilde{\rho}|, \quad x \in \mathbb{R} .
$$

The function $\rho \mapsto A(\rho, x)$ is piecewise linear for the sake of simplicity, but we can render it $C^{\infty}$ by a smoothing process. The lack of regularity of $\rho \mapsto A(\rho, x)$ is not a key point here. Let us now check that the spatial discontinuities of $A$ are BV. We have

$$
\operatorname{TV} . A(\rho, .)=\sum_{n=1}^{+\infty}\left|A\left(\rho, n^{+}\right)-A\left(\rho, n^{-}\right)\right|,
$$

where

$$
\left|A\left(\rho, n^{+}\right)-A\left(\rho, n^{-}\right)\right|= \begin{cases}\left|a_{n}(\rho)-\bar{a}_{n}(\rho)\right|, & n \text { even } \\ \left|\bar{a}_{n+1}(\rho)-a_{n-1}(\rho)\right|, & n \text { odd }\end{cases}
$$

Case $1: \rho>2$. We have $\left|a_{n+1}(\rho)-\bar{a}_{n-1}(\rho)\right|=\frac{2}{3}\left(\rho+\frac{1}{n+1}\right)-\frac{2}{3}\left(\rho+\frac{1}{n-1}\right)$, and $\left|a_{n}(\rho)-\bar{a}_{n}(\rho)\right|=0$, then

$$
\operatorname{TV} . A(\rho, .)=\sum_{\substack{n=3 \\ n \text { odd }}}^{+\infty}\left|\frac{2}{3}\left(\rho+\frac{1}{n+1}\right)-\frac{2}{3}\left(\rho+\frac{1}{n-1}\right)\right| \leq \frac{4}{3} \sum_{\substack{n=3 \\ n \text { odd }}}^{+\infty} \frac{1}{(n-1)(n+1)}=\frac{1}{3}
$$

Case 2 : There exists $m \in \mathbb{N}$ such that $\rho \in\left(\frac{2}{m+1}, \frac{2}{m}\right]$.

$\operatorname{TV} . A(\rho,)=.\sum_{n=1}^{\left\lfloor\frac{m}{4}\right\rfloor}\left|A\left(\rho, n^{+}\right)-A\left(\rho, n^{-}\right)\right|+\sum_{n=\left\lfloor\frac{m}{4}\right\rfloor+1}^{m}\left|A\left(\rho, n^{+}\right)-A\left(\rho, n^{-}\right)\right|+\sum_{n=m+1}^{+\infty}\left|A\left(\rho, n^{+}\right)-A\left(\rho, n^{-}\right)\right|$ 
We start by estimating the third term. For $n \geq m+1$, we have $\frac{1}{2 n} \leq \frac{2}{n} \leq \frac{2}{m+1} \leq \rho$, and then $\left(a_{n}(\rho)-\bar{a}_{n}(\rho)\right)=0$. The third term is then equal to

$$
\begin{aligned}
\sum_{n=m+1}^{+\infty}\left|A\left(\rho, n^{+}\right)-A\left(\rho, n^{-}\right)\right|=\sum_{\substack{n=m+1 \\
n \text { odd }}}^{+\infty}\left|A\left(\rho, n^{+}\right)-A\left(\rho, n^{-}\right)\right| & =\sum_{\substack{n=m+1 \\
n \text { odd }}}^{+\infty}\left|\frac{2}{3}\left(\rho+\frac{1}{n+1}\right)-\frac{2}{3}\left(\rho+\frac{1}{n-1}\right)\right| \\
& \leq \frac{4}{3} \sum_{n=2}^{+\infty} \frac{1}{(n-1)(n+1)}<\frac{2 \pi^{2}}{9}
\end{aligned}
$$

We deal with the first term the following way. For $n \leq \frac{m}{4}$, we have $\rho \leq \frac{2}{m} \leq \frac{1}{2 n}$ which implies $\left|A\left(\rho, n^{+}\right)-A\left(\rho, n^{-}\right)\right|=|2 \rho-\rho|=\rho$. Thus

$$
\sum_{n=1}^{\left\lfloor\frac{m}{4}\right\rfloor}\left|A\left(\rho, n^{+}\right)-A\left(\rho, n^{-}\right)\right|=\sum_{n=1}^{\left\lfloor\frac{m}{4}\right\rfloor} \rho \leq \frac{m}{4} \frac{2}{m}=\frac{1}{2} .
$$

Let us finally deal with the second term. We pick $n \in\left[\left\lfloor\frac{m}{4}\right\rfloor+1, m\right]$. We have

$$
\begin{aligned}
& \left|a_{n}(\rho)-\bar{a}_{n}(\rho)\right| \leq a_{n}(\rho)+\bar{a}_{n}(\rho) \leq 2 \rho+\frac{2}{3 n}+\rho+\frac{2}{3 n} \\
& \left|\bar{a}_{n+1}(\rho)-a_{n-1}(\rho)\right| \leq \bar{a}_{n+1}(\rho)+a_{n-1}(\rho) \leq 2 \rho+\frac{2}{3(n+1)}+\rho+\frac{2}{3(n-1)}
\end{aligned}
$$

We get then, after separating the even and odd $n$

$$
\sum_{n=\left\lfloor\frac{m}{4}\right\rfloor+1}^{m}\left|A\left(\rho, n^{+}\right)-A\left(\rho, n^{-}\right)\right|=\sum_{\substack{n=\left\lfloor\frac{m}{4}\right\rfloor+1 \\ n \text { even }}}^{m}\left(3 \rho+\frac{4}{3 n}\right)+\sum_{\substack{n=\left\lfloor\frac{m}{4}\right\rfloor+1 \\ n \text { odd }}}^{m}\left(3 \rho+\frac{2}{3(n+1)}+\frac{2}{3(n-1)}\right) .
$$

Let us focus on the term corresponding to $n$ even. On the first hand, since $\rho \leq \frac{2}{m}$ we have

$$
\sum_{\substack{n=\left\lfloor\frac{m}{4}\right\rfloor+1 \\ n \text { even }}}^{m} 3 \rho \leq 3\left(m-\frac{m}{4}-1\right) \frac{2}{m} \leq \frac{9}{2}
$$

and on the other hand, for $m>4$

$$
\sum_{\substack{n=\left\lfloor\frac{m}{4}\right\rfloor+1 \\ n \text { even }}}^{m} \frac{4}{3 n} \leq \frac{4}{3} \int_{\left\lfloor\frac{m}{4}\right\rfloor}^{m} \frac{d x}{x}=\frac{4}{3}\left(\log (m)-\log \left(\left\lfloor\frac{m}{4}\right\rfloor\right)\right) \leq \frac{4}{3} \log \left(\frac{4 m}{m-4}\right) \leq \frac{4}{3} \log (20) .
$$

The sum is trivially finite for $m=1,2,3$ or 4 . The terms corresponding to $n$ odd are treated the same way. As a consequence, the second line of $(\mathrm{H}-1)$ is satisfied.

2. The flux violates assumption (9). Using the expression (24) for the initial datum

$$
\begin{gathered}
\sum_{n=1}^{+\infty}\left|A\left(\rho_{0}\left(n^{+}\right), n^{+}\right)-A\left(\rho_{0}\left(n^{-}\right), n^{-}\right)\right|=\sum_{n=1}^{+\infty}\left|A\left(\frac{1}{n}, n^{+}\right)-A\left(\frac{1}{n}, n^{-}\right)\right| \\
=\sum_{\substack{n=1 \\
n \text { even }}}^{+\infty}\left|a_{n}\left(\frac{1}{n}\right)-\bar{a}_{n}\left(\frac{1}{n}\right)\right|+\sum_{\substack{n=1 \\
n \text { odd }}}^{+\infty}\left|\bar{a}_{n+1}\left(\frac{1}{n}\right)-a_{n-1}\left(\frac{1}{n}\right)\right|
\end{gathered}
$$



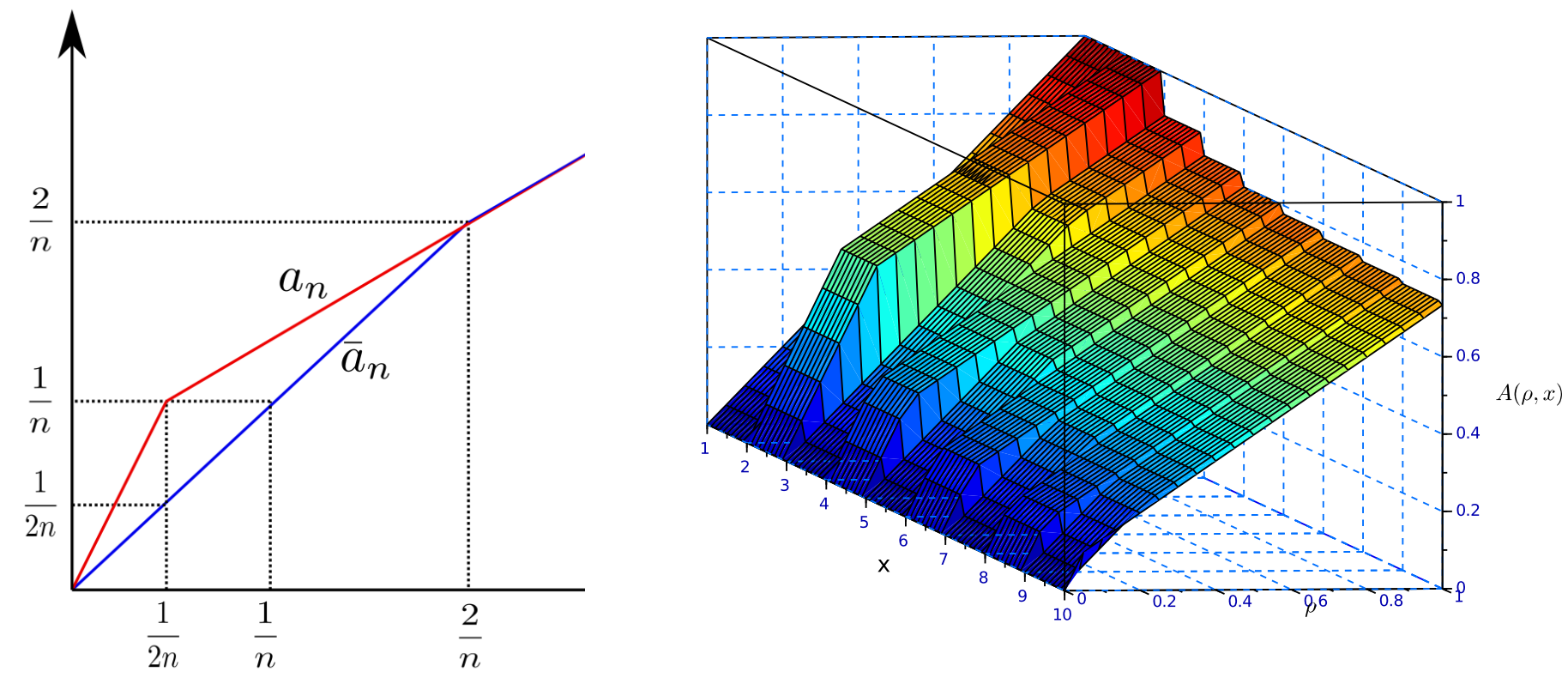

Figure 4: Left: The flux $A(., x)$ depends on $x$. For $x \in[n-1, n)$ with $\mathrm{n}$ odd, for $\rho \in\left(0, \frac{2}{n}\right)$, $A(\rho, x)=\bar{a}_{n}(\rho)=\rho$, for $\rho \in\left(\frac{2}{n},+\infty\right), A(\rho, x)=\bar{a}_{n}(\rho)=\frac{2}{3}\left(\rho+\frac{1}{n}\right)$. For $x \in[n, n+1)$ with n even, for $\rho \in\left(0, \frac{1}{2 n}\right), A(\rho, x)=a_{n}(\rho)=2 \rho$, and for $\rho \in\left(\frac{1}{2 n},+\infty\right), A(\rho, x)=a_{n}(\rho)=\frac{2}{3}\left(\rho+\frac{1}{n}\right)$. Right: Tri-dimensional visualization of the flux function $A$ detailed in the counter-example.

Using now expression (25), we find that the terms corresponding to the even integers are giving the sum

$$
\sum_{\substack{n=1 \\ n \text { even }}}^{+\infty}\left|\frac{2}{3}\left(\frac{1}{n}+\frac{1}{n}\right)-\frac{1}{n}\right|=\frac{1}{3} \sum_{\substack{n=1 \\ n \text { even }}}^{+\infty} \frac{1}{n}=+\infty,
$$

and this ends the proof of Theorem (9).

Remark 2. We can assume the discontinuity points lay on a compact set, for example Disc $(A) \subset$ $\{\arctan (n), n \in \mathbb{N}\}$. In this counter example, there is no $R$ continuous such that $(\overrightarrow{\mathrm{H}-2})$ is satisfied (Indeed $R$ would blow up at $\rho=0$ ). We point out that $\rho=0$ does not play a specific role here, and that we can shift the flux so that the blow up for $R$ occurs at any $\rho>0$.

\section{Conclusion}

We focus on a spatially heterogeneous conservation law, and define a BV framework in which an adapted entropy solution defined by [10] exists for every Cauchy problem with BV initial datum. We also provide new BV estimates on the entropy solution. To prove our result, we introduce a key assumption on the flux, which is necessary in the following sense: we exhibit a flux that does not satisfy this assumption and an initial condition $\rho_{0} \in B V(\mathbb{R})$ such that the total variation of 
the solution $\rho(t,$.$) blows up instantaneously. If the variables of the flux are separated, or are a$ combination of separated variables, which is the focus of most authors, the key condition $(\mathrm{H}-2)$ is deduced from $\mathrm{H}-\mathrm{0}$ and $\mathrm{H}-1$.

\section{Appendix 1: Proof of Lemma 1.}

Proof. Let us first introduce some notations. For all $\rho>0$, we consider the following well-defined limits

$$
A\left(\rho, x^{-}\right)=\lim _{\substack{y \rightarrow x \\ y<x}} A(\rho, y), \quad A\left(\rho, x^{+}\right)=\lim _{\substack{y \rightarrow x \\ y>x}} A(\rho, y),
$$

and denote the jump of $A$ at $(\rho, x)$ by

$$
J_{\rho}(x)=\left|A\left(\rho, x^{+}\right)-A\left(\rho, x^{-}\right)\right| .
$$

We then write

$$
\operatorname{Disc}(A)=\bigcup_{N \in \mathbb{N}} \bigcup_{k \in \mathbb{N}} I_{k, N}
$$

where

$$
I_{k, N}=\left\{x \in \mathbb{R} \mid \exists \rho_{x} \in[0, N] \text { such that } J_{\rho_{x}}(x) \geq \frac{1}{k}\right\}
$$

We claim that for all $N \geq 0, k \geq 0, I_{k, N}$ is finite, which makes $\operatorname{Disc}(A)$ at most countable. Let us assume by contradiction that there exists $(k, N) \in \mathbb{N}^{2}$ such that $I_{k, N}$ is infinite. Let us first notice that we have

$$
\exists \epsilon>0 \mid \forall x \in I_{k, N},\left\{\left|\rho-\rho_{x}\right| \leq \varepsilon \Longrightarrow J_{\rho}(x) \geq \frac{1}{2 k}\right\} .
$$

Indeed, $\forall x \in I_{k, N}, \forall \rho \in \mathbb{R}^{+}$,

$$
\begin{aligned}
\frac{1}{k} \leq J_{\rho_{x}}(x) & \leq\left|A\left(\rho, x^{+}\right)-A\left(\rho_{x}, x^{+}\right)\right|+J_{\rho}(x)+\left|A\left(\rho, x^{-}\right)-A\left(\rho_{x}, x^{-}\right)\right| \\
& \leq 2 C\left|\rho-\rho_{x}\right|+J_{\rho}(x)
\end{aligned}
$$

and (26) holds for $\varepsilon=\frac{1}{4 C k}$. There exists an integer $i \in\{0, \cdots,\lfloor N / \varepsilon+1\rfloor\}$ such that the interval $[i \epsilon,(i+1) \varepsilon]$ contains an infinite number of $\rho_{x}$, for $x \in I_{k, N}$ (note that the corresponding $\rho_{x}$ may actually not be distinct). Then, for that $i$,

$$
\operatorname{TV} . A(i, .) \geq \sum_{\substack{x \in I_{k, N} \\ \rho_{x} \in[i \epsilon,(i+1) \varepsilon]}}\left|A\left(i, x^{+}\right)-A\left(i, x^{-}\right)\right|>\sum_{\substack{x \in I_{k, N} \\ \rho_{x} \in[i \epsilon,(i+1) \varepsilon]}} \frac{1}{2 k}=+\infty,
$$

which contradicts $(\mathrm{H}-1)$.

\section{Appendix 2: In Example a), the Panov formulation selects the con- stant solution.}

We prove here that for the flux and initial condition (8), the Panov criterion selects (at least) the constant solution $\rho(x, t)=\frac{1}{2}$. As explained in the Subsection 1.2 of the Introduction, example a), the flux (8) was modified so that it satisfies (B-1.). To do so, we replace it by

$$
A_{M}(\rho, x)= \begin{cases}a_{M}^{-}(\rho), & x<0 \\ a_{M}^{+}(\rho), & x>0\end{cases}
$$


with

$$
a_{M}^{-}(\rho)=\left\{\begin{array}{ll}
\frac{(\rho-1)^{2}}{2}, & -(M+1) \leq \rho \leq M, \\
h(\rho), & M \leq \rho \leq M+1,
\end{array} \quad a_{M}^{+}(\rho)= \begin{cases}f(\rho), & -(M+1) \leq \rho \leq-M \\
\frac{\rho^{2}}{2}, & |\rho| \leq M, \\
g(\rho), & M \leq \rho \leq M+1,\end{cases}\right.
$$

where $f, g, h$ are chosen such that both $a_{M}^{-}$and $a_{M}^{+}$are convex, and such that $A_{M}(M+1, x)=$ $A_{M}(-(M+1), x)=(M+2)^{2}$. Even though the newly built flux depends on $M$, we drop this dependence in the notation in this Appendix, and we prove that the selected solution does actually not depend on $M$ for $M>1 / 2$. Notice that we can impose $f(\rho) \leq \frac{(\rho-1)^{2}}{2}$ for $\rho \in(-(M+1),-M)$ and $h(\rho) \leq g(\rho)$ for $\rho \in(M, M+1)$. To check that $\rho(x, t)=\frac{1}{2}$ satisfies the Panov selection criterion (7), since the spatial derivative of the flux $A$ contains only a singular part $\mu_{k}^{s}$ (at $x=0$ ), we need to evaluate the entropy dissipation, i.e. to check that the quantity

$$
\begin{aligned}
E(k) & =\frac{\partial}{\partial t}\left|\frac{1}{2}-k\right|+\frac{\partial}{\partial x}\left(\operatorname{sign}\left(\frac{1}{2}-k\right)\left(\frac{1}{8}-A(k, x)\right)\right)+\left|\mu_{k}^{s}(x)\right| \\
& =\frac{\partial}{\partial x}\left(\operatorname{sign}\left(\frac{1}{2}-k\right)\left(\frac{1}{8}-A(k, x)\right)\right)+\left|\mu_{k}^{s}(x)\right|,
\end{aligned}
$$

where

$$
\mu_{k}^{s}(x)=\frac{\partial}{\partial x} A(k, x)
$$

is non-positive for $k \in(-(M+1), M+1)$. We distinguish different cases depending on $k$.

Case 1. $k \in(-M, M)$. In that case, we have

$$
\frac{\partial}{\partial x} A(k, x)=\left(k-\frac{1}{2}\right) \delta_{x=0}
$$

and for $k<\frac{1}{2}$,

$$
\operatorname{sign}\left(\frac{1}{2}-k\right)\left(\frac{1}{8}-A(k, x)\right)=\left\{\begin{array}{l}
\frac{1}{8}-\frac{(k-1)^{2}}{2}, \quad x<0, \\
\frac{1}{8}-\frac{k^{2}}{2}, \quad x>0
\end{array}\right.
$$

and thus

$$
\frac{\partial}{\partial x}\left(\operatorname{sign}\left(\frac{1}{2}-k\right)\left(\frac{1}{8}-A(k, x)\right)\right)=\left(-\frac{k^{2}}{2}+\frac{(k-1)^{2}}{2}\right) \delta_{x=0}=\left|k-\frac{1}{2}\right| \delta_{x=0} .
$$

For $k>\frac{1}{2}$

$$
\operatorname{sign}\left(\frac{1}{2}-k\right)\left(\frac{1}{8}-A(k, x)\right)=\left\{\begin{array}{l}
-\frac{1}{8}+\frac{(k-1)^{2}}{2}, \quad x<0 \\
-\frac{1}{8}+\frac{k^{2}}{2}, \quad x>0
\end{array}\right.
$$

and thus

$$
\frac{\partial}{\partial x}\left(\operatorname{sign}\left(\frac{1}{2}-k\right)\left(\frac{1}{8}-A(k, x)\right)\right)=\left(\frac{k^{2}}{2}-\frac{(k-1)^{2}}{2}\right) \delta_{x=0}=\left|k-\frac{1}{2}\right| \delta_{x=0}
$$


This implies that $E(k)=0$.

Case 2. $k \in(-(M+1),-M)$. In that case, we have

$$
\left|\frac{\partial}{\partial x} A(k, x)\right|=\left|f(k)-\frac{(k-1)^{2}}{2}\right| \delta_{x=0}=\left(\frac{(k-1)^{2}}{2}-f(k)\right) \delta_{x=0},
$$

and

$$
\frac{\partial}{\partial x}\left(\operatorname{sign}\left(\frac{1}{2}-k\right)\left(\frac{1}{8}-A(k, x)\right)\right)=\left(\frac{(k-1)^{2}}{2}-f(k)\right) \delta_{x=0},
$$

so that $E(k)=0$.

Case 3. $k \in(M, M+1)$. Similarly, we have $E(k)=0$ since

$$
\left|\frac{\partial}{\partial x} A(k, x)\right|=|g(k)-h(k)| \delta_{x=0}=(g(k)-h(k)) \delta_{x=0},
$$

and

$$
\frac{\partial}{\partial x}\left(\operatorname{sign}\left(\frac{1}{2}-k\right)\left(\frac{1}{8}-A(k, x)\right)\right)=(h(k)-g(k)) \delta_{x=0} .
$$

\section{References}

[1] Adimurthi, Rajib Dutta, Shyam Sundar Ghoshal, and G. D. Veerappa Gowda. Existence and nonexistence of TV bounds for scalar conservation laws with discontinuous flux. Comm. Pure Appl. Math., 64(1):84-115, 2011.

[2] Adimurthi, J. Jaffré, and G. D. Veerappa Gowda. Godunov-type methods for conservation laws with a flux function discontinuous in space. SIAM J. Numer. Anal., 42(1):179-208 (electronic), 2004 .

[3] B. Andreianov, K. Brenner, and C. Cancès. Approximating the vanishing capillarity limit of two-phase flow in multi-dimensional heterogeneous porous medium. ZAMM Z. Angew. Math. Mech., 94(7-8):655-667, 2014.

[4] B. Andreianov and C. Cancès. On interface transmission conditions for conservation laws with discontinuous flux of general shape. Journal of Hyperbolic Differential Equations, 12(02):343$384,2015$.

[5] B. Andreianov, P. Goatin, and N. Seguin. Finite volume schemes for locally constrained conservation laws. Numer. Math., 115(4):609-645, 2010. With supplementary material available online.

[6] B. Andreianov, K. H. Karlsen, and N. H. Risebro. A theory of $L^{1}$-dissipative solvers for scalar conservation laws with discontinuous flux. Arch. Ration. Mech. Anal., 201(1):27-86, 2011.

[7] E. Audusse and B. Perthame. Uniqueness for scalar conservation laws with discontinuous flux via adapted entropies. Proc. Roy. Soc. Edinburgh Sect. A, 135(2):253-265, 2005.

[8] B. Aymard, F. Clément, F. Coquel, and M. Postel. A numerical method for transport equations with discontinuous flux functions: application to mathematical modeling of cell dynamics. SIAM J. Sci. Comput., 35(6):A2442-A2468, 2013. 
[9] F. Bachmann and J. Vovelle. Existence and uniqueness of entropy solution of scalar conservation laws with a flux function involving discontinuous coefficients. Comm. Partial Differential Equations, 31(1-3):371-395, 2006.

[10] P. Baiti and H. K. Jenssen. Well-posedness for a class of $2 \times 2$ conservation laws with $L^{\infty}$ data. J. Differential Equations, 140(1):161-185, 1997.

[11] A. Bressan. Hyperbolic systems of conservation laws, volume 20 of Oxford Lecture Series in Mathematics and its Applications. Oxford University Press, Oxford, 2000. The one-dimensional Cauchy problem.

[12] M. Bulíček, P. Gwiazda, J. Málek, and A. Świerczewska-Gwiazda. On scalar hyperbolic conservation laws with a discontinuous flux. Math. Models Methods Appl. Sci., 21(1):89-113, 2011.

[13] R. Bürger, K. H. Karlsen, N. H. Risebro, and J. D. Towers. Well-posedness in $B V_{t}$ and convergence of a difference scheme for continuous sedimentation in ideal clarifier-thickener units. Numer. Math., 97(1):25-65, 2004.

[14] G. M. Coclite, M. Garavello, and B. Piccoli. Traffic flow on a road network. SIAM J. Math. Anal., 36(6):1862-1886, 2005.

[15] G. M. Coclite and N. H. Risebro. Conservation laws with time dependent discontinuous coefficients. SIAM J. Math. Anal., 36(4):1293-1309 (electronic), 2005.

[16] Graziano Crasta, Virginia De Cicco, and Guido De Philippis. Kinetic formulation and uniqueness for scalar conservation laws with discontinuous flux. Comm. Partial Differential Equations, 40(4):694-726, 2015.

[17] Graziano Crasta, Virginia De Cicco, Guido De Philippis, and Francesco Ghiraldin. Structure of solutions of multidimensional conservation laws with discontinuous flux and applications to uniqueness. Arch. Ration. Mech. Anal., 221(2):961-985, 2016.

[18] C. M. Dafermos. Hyperbolic conservation laws in continuum physics, volume 325 of Grundlehren der Mathematischen Wissenschaften [Fundamental Principles of Mathematical Sciences]. Springer-Verlag, Berlin, third edition, 2010.

[19] A.-L. Dalibard. Homogenization of non-linear scalar conservation laws. Arch. Ration. Mech. Anal., 192(1):117-164, 2009.

[20] M. Garavello, K. Han, and B. Piccoli. Models for Vehicular Traffic on Networks, volume 9 of AIMS Series on Applied Mathematics. American Institute of Mathematical Sciences (AIMS), 2016.

[21] M. Garavello, R. Natalini, B. Piccoli, and A. Terracina. A riemann solver approach for conservation laws with discontinuous flux. In Sylvie Benzoni-Gavage and Denis Serre, editors, Hyperbolic Problems: Theory, Numerics, Applications, pages 1029-1036. Springer Berlin Heidelberg, 2008.

[22] J. Jimenez. Mathematical analysis of a scalar multidimensional conservation law with discontinuous flux. J. Evol. Equ., 11(3):553-576, 2011.

[23] K. H. Karlsen, N. H. Risebro, and J. D. Towers. Upwind difference approximations for degenerate parabolic convection-diffusion equations with a discontinuous coefficient. IMA J. Numer. Anal., 22(4):623-664, 2002. 
[24] Christian Klingenberg and Nils Henrik Risebro. Convex conservation laws with discontinuous coefficients. Existence, uniqueness and asymptotic behavior. Comm. Partial Differential Equations, 20(11-12):1959-1990, 1995.

[25] S. N. Kružkov. First order quasilinear equations with several independent variables. Mat. Sb. (N.S.), 81 (123):228-255, 1970.

[26] Martin Lazar and Darko Mitrović. Existence of solutions for a scalar conservation law with a flux of low regularity. Electron. J. Differential Equations, pages Paper No. 325, 18, 2016.

[27] R. J. LeVeque. Finite volume methods for hyperbolic problems. Cambridge University Press, 2002 .

[28] E. Yu. Panov. Existence and strong pre-compactness properties for entropy solutions of a firstorder quasilinear equation with discontinuous flux. Arch. Ration. Mech. Anal., 195(2):643-673, 2010 .

[29] B. Perthame, N. Seguin, and M. Tournus. A simple derivation of BV bounds for inhomogeneous relaxation systems. Comm. Math. Sci., 13(2):577-586, 2015.

[30] F. Poupaud and M. Rascle. Measure solutions to the linear multi-dimensional transport equation with non-smooth coefficients. Comm. Partial Differential Equations, 22(1-2):337-358, 1997.

[31] N. Seguin and J. Vovelle. Analysis and approximation of a scalar conservation law with a flux function with discontinuous coefficients. Math. Models Methods Appl. Sci., 13(2):221-257, 2003.

[32] J. D. Towers. Convergence of a difference scheme for conservation laws with a discontinuous flux. SIAM J. Numer. Anal., 38(2):681-698 (electronic), 2000.

[33] A. Vasseur. Strong traces for solutions of multidimensional scalar conservation laws. Arch. Ration. Mech. Anal., 160(3):181-193, 2001. 